\title{
Sinopsis de los Hydrophiloidea de México (Coleoptera: Hydrophilidae, Helophoridae, Epimetopidae, Georissidae e Hydrochidae), con una clave para la identificación de los géneros
}

\author{
Synopsis of the Hydrophiloidea of Mexico (Coleoptera: Hydrophilidae, Helophoridae, \\ Epimetopidae, Georissidae, and Hydrochidae), with a key for the identification of genera
}

\author{
Roberto Arce- Pérez* y Miguel Ángel Morón \\ Instituto de Ecología, A.C. Km. 2.5 carr. Xalapa-Coatepec, 351, El Haya 91070 Xalapa, Veracruz, México. \\ *Correspondencia: roberto.arce@inecol.edu.mx
}

\begin{abstract}
Resumen. Se presentan una síntesis sobre la taxonomía, ecología y distribución de la superfamilia Hydrophiloidea en México, una clave para géneros y una lista para géneros y especies con información sobre los estados donde se han registrado. Los Hydrophiloidea están representados en México por 6 familias, 9 tribus, 36 géneros, 181 especies y 6 subespecies, que corresponden al 20\% de los géneros y $6 \%$ de las especies en el mundo, y al $95 \%$ y $67 \%$ respectivamente para Mesoamérica. Se registran por primera vez para el estado de Veracruz Oosternum attacomis Spangler, 1962 y Sphaeridium scarabaeoides Linneo, 1758. Los estados con mayor riqueza de especies son Veracruz (72), Oaxaca (50), Sinaloa (44) y Sonora (43).

Palabras clave: Coleoptera, Hydrophiloidea, México.

Abstract. Taxonomic, ecological and distributional information regarding Mexican Hydrophiloidea is summarized. A key to genera is added and a checklist of the genera and species as well as geographic records for each state are presented. Hydrophiloidea are represented in Mexico by 6 families, 9 tribes, 36 genera, 181 species and 6 subspecies, corresponding to $20 \%$ and $6 \%$ of world genera and species respectively. In total, $95 \%$ and $67 \%$ of the recorded Mesoamerican genera and species are currently known from Mexico. Oosternum attacomis Spangler, 1962 and Sphaeridium scarabaeoides Linneo, 1758 are recorded for the first time from Veracruz. The states with the highest number of recorded species are Veracruz (72), Oaxaca (50), Sinaloa (44), and Sonora (43).
\end{abstract}

Key words: Coleoptera, Hydrophiloidea, Mexico.

\section{Introducción}

Los Hydrophiloidea constituyen uno de los grupos más numerosos de coleópteros con hábitos acuáticos y semiacuáticos. Más de la mitad de sus especies están adaptadas a la vida en el agua, o al menos en biotopos marginales, a veces inundables y otras veces riparios. La mayoría de las especies son depredadoras en su estado larval, y primariamente detritívoras, fitófagas y necrófagas en el imaginal; pocas son nadadoras activas en su estado adulto, la mayoría se desplazan sobre sustratos vegetales o sustratos inertes sumergidos y las riparias sobre el sedimento. Las larvas de algunos géneros son bentónicas, otras utilizan permanentemente soportes vegetales sumergidos. La superfamilia está integrada por formas adultas de tamaño pequeño $(1 \mathrm{~mm})$

Recibido: 19 mayo 2010; aceptado: 14 0ctubre 2010 hasta relativamente grandes $(50 \mathrm{~mm})$; la forma del cuerpo varía en relación con sus hábitos, las especies nadadoras presentan un cuerpo oval en vista dorsal, con el dorso más o menos convexo pero siempre curvado, la superficie ventral plana, salvo por un proceso mesosternal saliente, o bien, una quilla mesometasternal (forma hidrodinámica), además de presentar tibias y tarsos medios y posteriores aplanados y orlados con sedas para la natación (Berosini, Hydrophilini: Hydrophilina); las formas que son incapaces de nadar, fácilmente caminan y se mueven bajo el agua sobre sustratos vegetales, arenosos o lodosos, y generalmente son de forma ancha y convexa dorsalmente, aunque hay otras más bien anchas y planas, mientras que la superficie ventral es cóncava o ligeramente plana; en este caso, las patas no están modificadas para la natación (Anacaenini, Chatarthriini, Hydrophilini: Acidocerina); las formas riparias están asociadas a la vegetación y sedimentos de la orilla de los cuerpos de agua, y presentan un cuerpo de forma robusta, alargada y/o gibosa, con patas robustas e 
hileras de espinas tibiales y tarsos cortos, y generalmente están cubiertas por partículas del sedimento, barro, lodo o arena (Georissidae, Epimetopidae, Helophoridae, en parte). Las formas de vida acuática presentan una vestidura ventral hidrófoba que les permite retener una capa de aire mientras están sumergidas, la cual les confiere un brillo plateado; por ello también se les conoce como escarabajos plateados del agua.

En Hydrophilidae, los miembros de Sphaeridiinae, terrestres y/o semiacuáticos, son pequeños, ovales y convexos, con la superficie ventral cóncava o ligeramente plana; generalmente están asociados con sustratos muy húmedos. Las especies semiacuáticas también presentan vestidura hidrófoba, lo que sugiere que la modalidad de vida acuática se perdió secundariamente y/o que la humedad elevada de su hábitat habitual les exigió conservar esta adaptación especial. Carácter común en los adultos de toda la superfamilia es la conformación de la antena, rematada en una maza pubescente formada por 3 artejos (4 en Sperpcheus); los colores predominantes son pardo-negruzco con iridiscencias metálicas, pasando por pardo-amarillento o rojizo, hay manchados (maculatus) o bandeados (vittatus) con entremezcla de colores amarillo, verde, azul o cobrizo; la superficie puede ser lisa, granulosa, con fosetas, surcos, carinas y/o tubérculos. Las larvas en general son campodeiformes, alargadas, cilíndricas o cónicas con patas desarrolladas y una longitud de 4-60 mm, de color pardogrisáceo o amarillo- rojizo.

La gran mayoría de las especies de hidrofiloideos viven dentro o a la orilla de los cuerpos de agua dulce y limpia; sin embargo, algunas soportan niveles de contaminación, otras habitan manantiales, ambientes salinos y aun la zona intertidal de las playas rocosas, mientras que los adultos terrestres y semiacuáticos de la subfamilia Sphaeridiinae habitan en sitios húmedos, tales como hongos en descomposición, algas, estiércol, nidos de hormigas, bajo la corteza podrida de los troncos, en nidos y desechos de aves y mamíferos marinos, en el agua que se deposita en las axilas de las hojas de plantas, tales como Araceae y Marantaceae entre otras. En varias obras se ha discutido la limitación del nombre común "coleópteros basureros del agua", pues con muy raras excepciones las larvas son voraces depredadoras y los adultos se alimentan con algas, hongos, hojas en descomposición u otra vegetación; ocasionalmente devoran los tejidos de animales muertos, otros son detritívoros o depredadores, por lo que quizá el nombre más adecuado para la superfamilia sería "coleópteros omnívoros del agua" (Leech y Chandler, 1956; Spangler, 1982; Van Tassell, 2000; Oliva et al., 2002).

Algunas especies de Hydrophilidae llegan a tener importancia médica y económica: los adultos y larvas de Hydrophilus triangularis Say, 1823, Tropisternus lateralis nimbatus (Say, 1823) y Berosus infuscatus Say, 1823 han sido registrados como depredadores de larvas de mosquitos Culex quinquefasciatus Say, 1826 y Aedes taeniorhynchus (Wiedemann, 1821). Por lo menos 3 especies de Dactylosternum se han utilizado para control del escarabajo barrenador de la caña de azúcar en Filipinas y Hawaii, y una más para el control del escarabajo barrenador del plátano en Jamaica. En otros casos se han registrado especies de Helophoridae, tanto larvas como adultos, atacando cultivos de nabo, repollo y otras crucíferas, además de trigo y pastos, y adultos de Hydrophilus triangularis como plaga en criaderos de peces. Tanto los adultos como las larvas de Hydrophiloidea intervienen en varios niveles de la red trófica; como desintegradores aceleran el flujo de materia en los ambientes acuáticos y riparios; controlan poblaciones de invertebrados, así como de juveniles de peces y anfibios; además constituyen el alimento de peces, anfibios, reptiles, aves y mamíferos acuáticos y terrestres (Leech y Chandler, 1956; Nelson, 1977; Spangler, 1982; Van Tassell, 2000).

Taxonómicamente el grupo fue tratado como una familia por Leech y Chandler (1956), Arnett (1972), Spangler (1982, 1991), Smetana (1988), Newton y Thayer (1992) y Lawrence y Newton (1995). Sin embargo, diferentes autores, como Hansen (1991, 1995), que usando caracteres de los adultos, Beutel (1994), que emplea los de la cápsula cefálica de larvas y adultos, y Archangelsky (1998), los del preimago, proponen la elevación de las subfamilias al rango de familias (Helophoridae, Epimetopidae, Georissidae, Hydrochidae, Spercheidae e Hydrophilidae), propuesta que se sigue en este trabajo. Zaitzev en 1908 (citado por Hansen, 1999), en su catálogo de familias de coleópteros acuáticos, reconoce 114 géneros y 1336 especies dentro de la familia Hydrophilidae; ahora, un siglo después, se registran en el mundo 8 familias, 181 géneros y 3151 especies (Hansen, 1999; Short y Hebauer, 2006).

El objetivo del presente trabajo es proporcionar una síntesis sobre la taxonomía, biología y distribución en México de las especies de la superfamilia Hydrophiloidea de utilidad para futuros trabajos sobre sistemática, biología y zoogeografía del grupo. Estos datos contribuirán a los estudios sobre la conservación de los ambientes acuáticos epicontinentales.

\section{Materiales y métodos}

El conocimiento sobre la superfamilia Hydrophiloidea de México está basado en revisiones para América del Norte (Arnett, 1972; Pennak, 1978; White y Brigham, 1996), trabajos regionales de los Estados Unidos (Young, 1954; Leech y Chandler, 1956; Hatch, 1965), y revisiones genéricas para las regiones neártica (Miller, 1974; Gundersen 1977, 1978; 
Smetana, 1974, 1978, 1980, 1984, 1985a) y neotropical (Short y Perkins, 2004; Short, 2005b; Komarek, 2005; Short y Torres, 2006; Firàcek y Short, 2006). Además, en obras antiguas para Mesoamérica y México (Sharp, 1882, 1887; Blackwelder, 1944; Leech, 1948) y en la descripción de especies nuevas (Wooldridge, 1969, 1973; Smetana, 1978; Spangler y Santiago-Fragoso, 1979; Santiago-Fragoso y Mejorada-Gómez, 1995; Short, 2005a). Aun cuando esta literatura es una excelente ayuda, es insuficiente para realizar estudios detallados, sobre todo porque no contienen claves taxonómicas para la mayoría de los géneros y especies de las familias presentes en México.

En este trabajo se elabora una clave (basada en Hansen, 1991 y Van Tassell, 2000) para las familias, tribus y géneros conocidos de México, asi como una lista de especies con la siguiente información: especie, autor, año, página de la descripción original, distribución conocida en México y referencias. El número entre paréntesis después del mes de recolección indica el número de adultos. En la región de la sierra de Santa Marta, Veracruz, México, se realizó un estudio prospectivo para conocer la biodiversidad del suelo en cultivos de maíz, terrenos agroforestales, pastizales y bosque tropical lluvioso. Empleando trampas de pozo y la técnica de monolitos de suelo (Anderson e Ingram, 1993) se obtuvieron ejemplares de Sphaeridiinae (Hydrophilidae) cuyos registros precisos se incorporaron a la lista, además de otros ejemplares de la misma subfamilia recolectados en estiércol de caballo en las Vigas de Ramírez, Veracruz. El material está depositado en la colección entomológica del Instituto de Ecología, A.C., Xalapa, Ver., México (IEXA).

\section{Resultados}

Para América del Norte al norte de México se registraron 37 géneros y 315 especies (Van Tassell, 2000), de Mesoamérica 279 especies en 38 géneros (Spangler, 1982; Short y Hebauer, 2006), y de México 27 géneros y 129 especies (Arce-Pérez, 1995). Con la presente recopilación basada en los trabajos de Hansen (1999) y Short y Hebauer (2006) se confirma que la superfamilia Hydrophiloidea está representada en México por 5 familias, 9 tribus, 36 géneros, 181 especies y 6 subespecies, que corresponden al $20 \%$ de los géneros y al $6 \%$ de las especies del total en el mundo, y al 95\% y $67 \%$ respectivamente para Mesoamérica. Se les ha encontrado en todo México, y los estados con mayor número de especies registradas son Veracruz (72), Oaxaca (50), Sinaloa (44) y Sonora (43), y seguramente en el futuro se incrementará el número de especies, cuando se realice un mayor esfuerzo en recolectar y conocer este grupo de coleópteros acuáticos. Oosternum attacomis Spangler, 1962 y Sphaeridium scarabaeoides Linneo, 1758 se registran por primera vez para el estado de Veracruz y se corrobora la presencia estatal de Phaenonotum exstriatum (Say), 1835 y Cercyon variegatus Sharp, 1882 , cuyos datos nuevos se incorporaron al listado que se

presenta a continuación de la clave.

Clave para los Hydrophiloidea de México (adaptada de Van Tassell, 2000 y Hansen 1991).

1 Ojos parcial o totalmente divididos por canto ocular; primer esternito visible muy corto; pronoto proyectado hacia adelante y por encima de la mitad basal de la cabeza (Fig. 1)........Epimetopidae......................... Epimetopus

1' Ojos no divididos por el canto ocular; primer esternito bien desarrollado; pronoto proyectado o no............................2

2(1') Pronoto con amplios y profundos surcos longitudinales (Fig. 2) .....Helophoridae..........................Helophorus

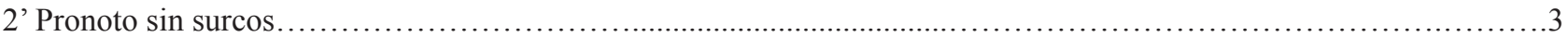

3(2') Pronoto con proyección que se extiende adelante y por encima de la cabeza (Fig. 3), metacoxas ampliamente separadas; procoxas y trocánteres fusionados para formar un plato que cubre el pequeño prosternón. Georissidae.

3' Pronoto sin una proyección como la de arriba, metacoxas no ampliamente separadas, procoxas y trocánteres no fusionados; prosternón bien desarrollado.

4(3') Pronoto distintivamente más angosto que la base de los élitros, superficie con elevaciones y depresiones poco profundas; escutelo muy pequeño y ojos protuberantes (Fig. 4)......Hydrochidae...............................Hydrochus

4' Pronoto casi, o tan ancho como la base de los élitros, superficie usualmente lisa escutelo grande y triangular; ojos prominentes o no......

5(4’) Antenas más cortas o subiguales en longitud a palpos maxilares; tarsómero basal de patas medias y posteriores más corto que el segundo o diminuto (Fig. 5) (excepto en Cymbiodyta, donde el segmento basal ha desaparecido); especies acuáticas......Hydrophilidae, Hydrophilinae......................................................... 6

5' Antenas usualmente más largas que los palpos maxilares; tarsómero basal de patas medias y posteriores más largo que 
el segundo; especies semiacuáticas o terrestres......Hydrophilidae, Sphaeridiinae...............................22

6(5) Primer esternito con excavación a cada lado de la línea media y cubiertas por densa franja de sedas duras y

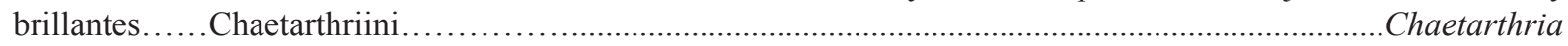

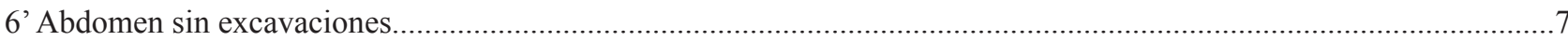

7(6') Cabeza fuertemente dirigida hacia abajo (flexionada) (Fig. 6); antenas usualmente con 7 artejos; escutelo largo y triangular; tibias medias y posteriores con hileras de sedas largas......Berosini............................. 8

7’ Cabeza no o sólo moderadamente flexionada (Fig. 7); antenas con 9 segmentos; escutelo no o escasamente más largo

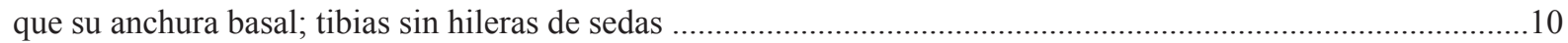

8(7) Ojos muy protuberantes; protibias delgadas y lineales; labro prominente; cuerpo de color testáceo-rojizo o amarillento, usualmente con reflexiones metálicas y puntuación o marcas negras; longitud del cuerpo mayor de $2 \mathrm{~mm}$...............9

8' Ojos no protuberantes; protibias triangulares, ensanchándose gradualmente hacia el ápice; labro muy corto; cuerpo de color negro y sin marcas; longitud $2 \mathrm{~mm}$ o menos ....................................................................................

9(8) Superficie ventral con vestidura aterciopelada que oscurece el integumento; protarsos del macho siempre con 5 artejos, segundo ligeramente ensanchado; mesosternón con cresta media de forma romboide y excavada

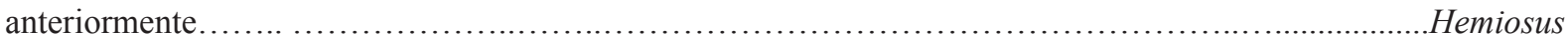

9' Superficie ventral con vestidura pero no aterciopelada que oscurezca el integumento; protarsos del macho siempre con 4 artejos, los 2 primeros claramente ensanchados (Fig. 8); mesosternón usualmente con cresta aguda como cuchilla que se extiende anteriormente.

Berosus

10(7') Abdomen con 5 esternitos (algunos Laccobius con 6), margen posterior del quinto esternito redondeado y sin escotadura apical; procesos meso y metasternales separados y no formando quilla esternal; segmento apical de palpos maxilares más largo que el penúltimo; profémures con vestidura basal, meso y metafémures glabros........

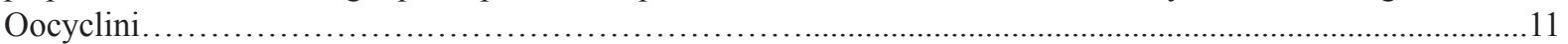

$10^{\prime}$ Abdomen de 5 esternitos, margen posterior del quinto esternito con pequeña escotadura apical bordeada de sedas rígidas amarillentas; si no, procesos meso y metasternal formando quilla continua; segmento apical de palpos maxilares más corto que penúltimo; meso y metafémures con vestidura basal que puede ser angosta...................12

11(10) Abdomen con 6 esternitos; trocánteres posteriores grandes, 1/3 la longitud de los fémures y ligeramente proyectados (Fig. 9); tibias posteriores arqueadas..

11' Abdomen con 5 esternitos; trocánteres posteriores de tamaño moderado y no proyectados apicalmente; tibias posteriores rectas Oocyclus

12(10') Cabeza, pronoto y élitros con series de puntos o por lo menos en alguna de estas partes (Figs. 10-12), especies

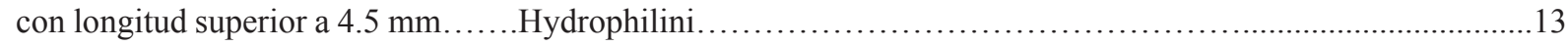

12' Cabeza pronoto y élitros usualmente sin series de puntos (escasos y muy diminutos), especies con longitud menor a

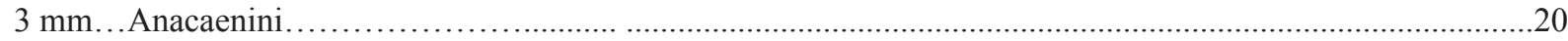

13(12) Meso y metasternón con quilla media que normalmente se extiende entre y más allá de las coxas posteriores como

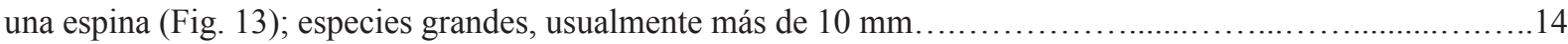

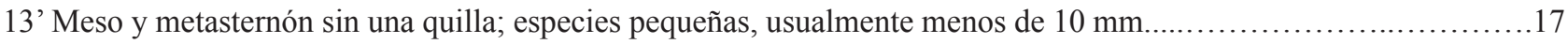

14(13) Prosternón ampliamente surcado para recibir la parte anterior de la quilla mesosternal (Fig. 13); quilla metasternal proyectándose como una espina más allá de los trocánteres posteriores

14' Prosternón carinado (Fig. 14), no surcado; quilla metasternal no o sólo ligeramente rebasando los trocánteres posteriores

15(14) Especies grandes, 30-45 mm de longitud; palpo maxilar apical más corto que el penúltimo................Hydrophilus

15'Especies medianas, 6-15 mm de longitud; palpo maxilar apical igual o más largo que el penúltimo..........Tropisternus

16(14') Margen frontal del clípeo simple, truncado o arqueado (Fig. 15); maza antenal con los 2 primeros segmentos muy similares (Fig. 16) ........................................................................................................Hydrochara

16' Margen frontal del clípeo arqueado y emarginado mostrando el preclípeo (Fig. 17); primer artejo de maza antenal más amplio y diferente al segundo (Fig. 18) .............................................................................

17(13') Labro oculto bajo el clípeo que se extiende hacia afuera sobre la anchura de los ojos; antenas de 8 artejos; élitros con márgenes ampliamente expandidos (Fig. 19)......................................................Helobata

17' Labro totalmente expuesto, clípeo no extendido lateralmente; antenas de 9 artejos; élitros con márgenes no expandidos

18(17') Mesosternón con débil protuberancia o elevación piramidal, nunca una lámina longitudinal; élitros con hileras longitudinales de puntos; tarsos de 5 artejos, el basal pequeño; palpos maxilares con segmento pesudobasal curvado 
hacia fuera. Helochares

18' Mesosternón con una variada proyección laminar longitudinal o transversal; élitros confusamente punteados; tarsos de 4 o 5 artejos; palpos maxilares con segmento pesudobasal no curvado hacia fuera............................19

19(18') Tarsos medios y posteriores de 4 artejos; palpos maxilares uniformemente curvos hacia afuera (Fig. 20); mesosternón con cresta transversa (Fig. 21)......

Cymbiodyta

19' Tarsos medios y posteriores de 5 artejos, segundo usualmente diminuto; palpos maxilares con segundo artejo curvado hacia línea media (Fig. 22); mesosternón con lamina media longitudinal que a menudo presenta pequeño diente anterior

Enochrus

20(12') Élitros con estría sutural en mitad posterior; ojos no o sólo ligeramente emarginados 21

20’ Élitros sin estría sutural; ojos marcadamente emarginados anteriormente.

Notionotus

21(20) Prosternón longitudinalmente carinado a la mitad; metafémures sin vestidura hidrófoba (si está presente sólo restringida a la base); mesosternón sin elevación angular o protuberancia dentiforme anterior a coxas medias.

Paracymus

21 ' Prosternón no carinado, pero puede ser ligeramente convexo a la mitad; metafémures usualmente con vestidura hidrófoba extendida; mesosternón con elevación angular prominente o protuberancia dentiforme anterior a coxas medias (Fig. 23).

Anacaena

22(5') Cabeza ancha, no marginada frente a los ojos, base de antenas ocultas por expansiones laterales (Fig. 24); primer esternito tan largo como el segundo o ligeramente más corto

22'. Cabeza angostada o emarginada en frente de los ojos, exponiendo base de antenas (Fig. 25); primer esternito mucho más largo que el segundo.

23 (22) Maza antenal claramente compacta (Fig. 26); labro totalmente expuesto y bien pigmentado y esclerosado; escutelo mucho más largo que ancho; primer artejo de los tarsos medios y posteriores mucho más largo que el segundo; tibias con largas espinas en la cara exterior; ojos profundamente emarginados anteriormente Sphaeridiini.....Sphaeridium

23’ Maza antenal no compacta (Fig. 27), si lo es, entonces el labro casi cubierto por clípeo y blando y pálido, escutelo

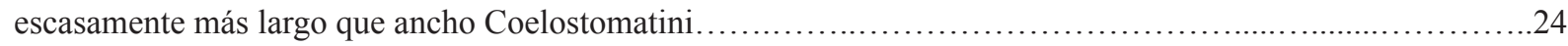

24(23') Maza antenal claramente compacta, élitros con estría sutural y 10 filas de perforaciones seriales; primer esternito carinado.

Dactylosternum

24' Maza antenal no compacta, élitros sin estría sutural ni filas de perforaciones seriales; primer esternito no carinado o

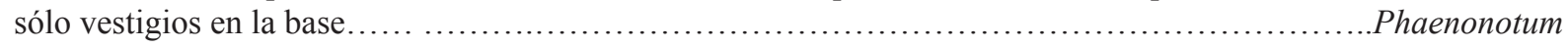

25(22'). Clípeo fuertemente dirigido hacia abajo y algo convexo visto en perfil (Fig. 6); labro largo y visible; epipleura y pseudoepipleura bien desarrolladas cerca del abdomen Omicrini.......................................

25'. Clípeo no dirigido hacia abajo, débilmente convexo en perfil (Fig. 28); labro oculto por el clípeo; epipleura no visible y psedoepipleura sólo ligeramente cerca del abdomen Megasternini........................................28

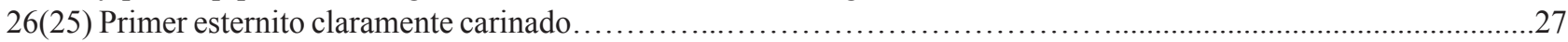

26' Primer esternito no carinado, prosternón claramente visible enfrente de procoxas (Fig. 29); antenas de 8 artejos, cuerpo alargado y oval poco convexo.

..Heteryon

27(26) Mesocaxas ampliamente separadas; proceso mesosternal suavemente inclinado lateralmente hacia abajo, no en un solo plano.

Omicrus

27' Mesocoxas angostamente separadas; proceso mesosternal plano y pentagonal; prosternón extremadamente corto, reducido a una angosta barra vertical frente de procoxas (Fig. 30); cuerpo fuertemente convexo.

28(25') Prosternón con largos y profundos surcos antenales que rebasan margen lateral del protórax (Fig. 31)................29

$28^{\prime}$ Prosternón con surcos antenales que no rebasan margen lateral del protórax (Fig. 32) ...............................31

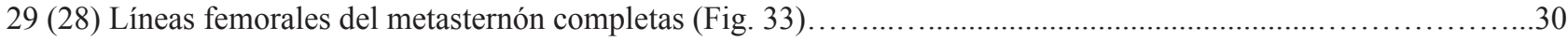

29'(28) Líneas femorales del metasternón reducidas o anterolateralmente vestigiales, primer esternito longitudinalmente carinado, margen externo de tibias anteriores fuertemente escotado (Fig. 34), prosternón elevado a la mitad para formar placa oval no carinada a la mitad ........................................................egasternum

30(29) Placa mesoesternal tan larga como ancha (Fig. 35), mesocoxas moderadamente separadas, cabeza y pronoto con microescultura en forma de líneas longitudinales; octava estría elitral delimitando de la tercera a séptima estrías posteriormente. .Cycrillum

30' Placa mesoesternal más ancha que larga (Fig. 36), mesocoxas ampliamente separadas; pronoto sin microescultura densa, placa prosternal pentagonal y mesosternón con líneas femorales (Fig. 33), primer esternito con 
carina

Cryptopleurum

31(28') Esquinas anterolaterales del metasterno claramente demarcadas del resto del metasternón por un reborde arqueado (Fig. 37). Élitros con 10 distintivas estrías; primer esternito con numerosos finos rebordes longitudinales a cada lado de la carina media. Oosternum

31' Metaesterno no como arriba o sólo con un ligero reborde cerca del margen posterior de las

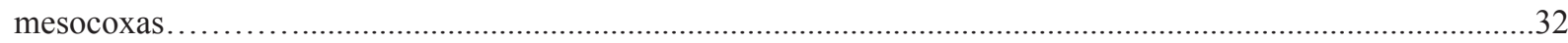

32(31') Margen anterior del prosternón con una incisión subrectangular media, cuyos márgenes laterales son rebordes proyectados (Fig. 38), élitros con fuertes estrías impresas y los intersticios levantados; elevaciones del mesometasternón algo cóncavas a la mitad.

Motonerus

32' Margen anterior del prosternón sin incisión, élitros con finas estrías y los intersticios planos, elevaciones del mesometasternales no como arriba.

33 (32’) Placa mesosternal de alargada a ovalada o casi lineal, estrecha anterior y posteriormente, con sus ápices redondeados y no toca porción levantada del metasternón (Fig. 39). Cercyon

33. Placa mesosternal pentagonal o con los lados paralelos, casi entre las mesocoxas, trunca posteriormente y tocando o fusionada con metasternón.

34(33'). Placa mesosternal tan larga como ancha..

34' Placa mesosternal 2 o 3 veces más larga que ancha (Fig. 40); mentón muy débilmente marginado anteriormente.

Pelosoma

35(34) El centro del prosternón tectiforme y fuertemente carinado a la mitad (Fig. 41). Surcos antenales débilmente definidos lateralmente Agna

35 El centro del prosternón bastante plano o finamente carinado (Fig. 42). Surcos antenales bien definidos por un reborde lateral. Deltostethus

Lista de las especies de Hydrophiloidea registradas para México

El 40\% de los datos se basa en obras para América del Norte y el norte de México, el 25\% corresponde a publicaciones sobre fauna mexicana, el 20\% en trabajos de México y América Central y el $15 \%$ en catálogos y trabajos de la región neotropical. La cita "Baja California" indica que no fue posible determinar si el registro pertenece al estado de Baja California (Norte) o a estado de Baja California Sur.

HYDROPHILOIDEA Latreille, 1802

1. HELOPHORIDAE Leach, 1815

1. Helophorus Fabricius, 1775

1. H. californicus Smetana, 1985a: 77

Distribución: Baja California y Durango. Adultos activos en abril (1), mayo (3) y junio (2) (Smetana, 1985a).

2. H. linearis Leconte, 1855: 357

Distribución: Baja California, Chiapas, Chihuahua, Distrito Federal, Durango, Estado de México, Guanajuato, Guerrero, Hidalgo, Jalisco, Oaxaca, Puebla, San Luis Potosí, Sonora y Zacatecas. Adultos activos en abril (128), junio (20), julio (103) y agosto (5), con registros de noviembre (16) y diciembre (8) (Campbell et al., 2008; Smetana, 1985a).

3. H. regularis Sharp, 1882: 89

Distribución: Guanajuato, Hidalgo, Jalisco, Michoacán, Oaxaca, San Luis Potosí, Sonora y Zacatecas. Adultos activos en julio (19), septiembre (1), noviembre (3), diciembre (3) (Sharp, 1882; Smetana, 1985a).

4. H. robertis Smetana, 1985a: 71

Distribución: Distrito Federal, Durango, Hidalgo, Jalisco, Puebla, San Luis Potosí y Zacatecas. Adultos activos en junio (172), julio (10), agosto (5), septiembre (1), noviembre (5), diciembre (1) (Smetana, 1985a).

2. EPIMETOPIDAE Zaitzev, 1908

2. Epimetopus Lacordaire, 1854

5. E. costatus (LeConte, 1874): 48

Distribución: "México" (necesita confirmación, Hansen, 1999; Lanzhu y Jäch, 1998; Perkins, 1979).

6. E. fisheri Perkins, 1979: 322

Distribución: Sonora, entre los 380 y $800 \mathrm{~m}$ de altitud. Adultos activos en agosto (9) y octubre (22) (Perkins, 1979).

7. E. punctipennis Perkins, 1979: 321

Distribución: Sonora, entre 50 y $800 \mathrm{~m}$ de altitud. Adultos activos en agosto (1), octubre (42) y noviembre (1) (Perkins, 1979).

8. E. thermarum Schwarz y Barber, 1918: 130

Distribución: Baja California Sur, entre los 200 y 400 m de altitud. Adultos activos en julio (16); fueron atraídos por trampa de luz (Lanzhu y Jäch, 1998; Leech, 1948b).

3. GEORISSIDAE Castelnau, 1840

3. Georissus Latreille, 1809

9. G. minor Sharp, 1882: 141 
Distribución: Estado de México, Sonora y Zacatecas. Adultos activos en Junio (51) y entre noviembre y febrero (5) (Cortés-Aguilar et al., 2004; Chandler, 1973; Hinton, 1933).

4. HYDROCHIDAE Thomson, 1859

4. Hydrochus Leach, 1817

10. H. bernardi Makhan, 1994: 42

Distribución: Nayarit, a una altitud de 730 m (Hansen, 1999).

11. H. elineae Makhan, 1994: 41

Distribución: Nayarit, a una altitud de 730 m (Hansen, 1999).

12. H. vanvondeli Makhan, 1994: 41

Distribución: Nayarit, a una altitud de 730 m (Hansen, 1999).

13. H. variolatus LeConte, 1852: 211

Distribución: Baja California, a una altitud de $30 \mathrm{~m}$. Adultos activos durante junio y agosto (34) (Leech, 1948b).

14. Hydrochus sp.

Distribución: Distrito Federal (Santiago-Fragoso y Mejorada-Gómez, 1999).

5. HYDROPHILIDAE Latreille, 1802

Hydrophilinae

Berosini

5. Berosus Leach, 1817

15. B. blechrus Leech, 1948a: 38

Distribución: Sinaloa y Sonora (Van Tassell 1966).

16. B. dolerosus Leech, 1948b: 433

Distribución: Baja California Sur. Adultos activos en junio (16) (Leech, 1948b).

17. B. gordito Spangler y Santiago, 1979: 333

Distribución: Durango, en altitudes de 1720 a 1970 m.

Adultos activos en junio (30) y agosto (4) (Spangler y Santiago, 1979).

18. B. gracilipes Sharp, 1882: 83

Distribución: Oaxaca (Sharp, 1882).

19. B. griseus Sharp, 1882: 80

Distribución: Oaxaca, Sinaloa, Tabasco y Veracruz (Sharp, 1882, 1887; Van Tassell, 1966).

20. B. hoplites Sharp, 1887: 765

Distribución: Chihuahua, Distrito Federal, Durango, Guanajuato, Estado de México, Michoacán, Morelos, Puebla, San Luis Potosí, Sonora y Zacatecas (Santiago-Fragoso y Sandoval-Manrique, 2001; Sharp, 1887; Van Tassell, 1966).

21. B. infuscatus LeConte, 1855: 365

Distribución: Baja California Sur (24 adultos en julio), Chiapas, Chihuahua, Durango, Nuevo León, Oaxaca, Puebla, Sinaloa, Sonora y Yucatán (Darlington, 1936; Leech, 1948b; Sharp, 1882; Van Tassell, 1966).

22. B. ingratus Orchymont, 1946: 10

Distribución: Chihuahua, Distrito Federal, Estado de
México, Michoacán, Morelos, Puebla y Querétaro. En Morelos, adultos activos en enero (252), febrero (43), marzo (224), mayo (250), julio (584), agosto (60), octubre (1325), noviembre (413) y diciembre (294), en estanques temporales con gramíneas emergentes, márgenes del río con fanerógamas acuáticas Ceratophyllum demersum, Ludwigia repens, y algas verdes que se desarrollan en los márgenes del río (Arce-Pérez, 1986; Arce-Pérez y NoveloGutiérrez, 1990; Hansen, 1999; Van Tassell, 1966).

23. B. maculosus Mannerheim, 1853: 167

Distribución: Baja California Sur (Van Tassell, 1966).

24. B. metalliceps Sharp, 1882: 83

Distribución: Nayarit, Durango, Nuevo León y Sinaloa. Localizados en aguas salobres (Leech, 1948b; Sharp, 1882; Van Tassell, 1966).

25. B. mexicanus Sharp, 1882: 82

Distribución: Colima, Distrito Federal, Guanajuato, Hidalgo, Jalisco, Estado de México, Michoacán, Morelos, Nayarit, Puebla, Querétaro y Sinaloa. En Morelos se recolectó en enero (30), febrero (1), marzo (13), abril (5), mayo (7), junio (2), noviembre (5) y diciembre (566) (Campbell et al., 2008; Hansen, 1999; Santiago-Fragoso y MejoradaGómez 1999; Santiago-Fragoso y Sandoval-Manrique. 2001; Sharp, 1882; Van Tassell, 1966).

26. B. miles LeConte, 1855: 363

Distribución: Baja California Sur (10 adultos activos en julio), Chihuahua, Nuevo León, Sonora y Zacatecas (Leech, 1948b; Van Tassell, 1966).

27. B. moerens Sharp, 1882: 84

Distribución: Baja California Sur (9 adultos activos en junio), Coahuila, Chihuahua, Guerrero, Hidalgo, Oaxaca, Puebla, San Luis Potosí, Sinaloa, Sonora y Veracruz (Campbell et al., 2008; Leech, 1948b; Sharp, 1882, 1887; Van Tassell, 1966).

28. B. notapeltatus Van Tassell, 1963: 1

Distribución: Estado de México (Van Tassell, 1966).

29. B. peregrinus (Herbst, 1797): 314

Distribución: Baja California, Coahuila, Chihuahua, Nuevo León y Sonora (Van Tassell, 1966).

30. B. puncticollis Sharp, 1882: 80

Distribución: Oaxaca (un adulto en junio), Sinaloa, Sonora y Veracruz (Sharp, 1882; Van Tassell, 1966).

31. B. punctatissimus LeConte, 1852: 211

Distribución: Baja California, Baja California Sur, Durango y Hidalgo. Los adultos están presentes en la península de Baja California en junio-agosto, en manantiales, estanques, arroyos y diques de la irrigación, así como en tanques de riego. (Arce-Pérez y Novelo-Gutiérrez, 1991; Campbell et al., 2008; Leech, 1948b; Van Tassell, 1966).

32. B. regius Knisch, 1922: 111

Distribución: Nayarit, Sinaloa y Veracruz (Van Tassell, 1966). 
33. B. rubellus Knisch, 1922: 120

Distribución: Estado de México y Guanajuato (Van Tassell, 1966).

34. B. rufrenus Orchymont, 1946: 3

Distribución: Nayarit y Sinaloa. Adultos activos en agosto (Van Tassell, 1966).

35. B. rugulosus Horn, 1873: 124

Distribución: Baja California, Baja California Sur, Guerrero, Jalisco, Morelos, Michoacán, Nayarit, Puebla, Sinaloa y Sonora. Adultos activos en enero (106), marzo (117), mayo (6), junio (9), julio (28), algunas veces en grandes cantidades julio (1744), agosto (1038), octubre (912), noviembre (236) y diciembre (766); en estanques temporales con gramíneas emergentes, márgenes del río con fanerógamas acuáticas Ceratophyllum demersum, Ludwigia repens, y algas verdes que se desarrollan en los márgenes del río (Arce-Pérez, 1986; Arce-Pérez y NoveloGutiérrez, 1990; Leech, 1945, 1948b; Van Tassell, 1966).

36. B. sharpi Zaitzev, 1908: 357

Distribución: Registrada para la ciudad de Chihuahua y ciudad de México (Hansen, 1999; Sharp, 1887).

37. B. salvini Sharp, 1882: 79

Distribución: Guanajuato, Guerrero, Jalisco, Estado de México, Michoacán, Nayarit, Oaxaca, Puebla, Sinaloa y Sonora (Hansen, 1999; Sharp, 1882, 1887; Van Tassell, 1966).

38. B. stramineus Knisch, 1922: 124

Distribución: Baja California Sur, Colima, Jalisco, Nayarit, Sinaloa y Sonora. Adultos activos en junio (4) y julio (2) (Leech, 1948b; Van Tassell, 1966).

39. B. stylifer Horn, 1873: 121

Distribución: Aguascalientes, Chihuahua, Durango, Distrito Federal, Estado México, Guanajuato, Nuevo León, Tabasco, Sonora y Zacatecas. (Santiago-Fragoso y Mejorada-Gómez, 1999; Sharp, 1882; Van Tassell, 1966).

40. B. truncatipennis Castelnau, 1840: 56

Distribución: Colima, Durango, Jalisco, Michoacán, Nayarit, Oaxaca, San Luis Potosí, Sinaloa, Veracruz, Tabasco y Yucatán (Darlington, 1936; Sharp, 1882, 1887; Van Tassell, 1966).

41. B. undatus (Fabricius, 1792): 185

Distribución: Baja California Sur, Jalisco, Oaxaca, Sinaloa y Sonora (Sharp, 1887; Van Tassell, 1966).

6. Derallus Sharp, 1882

42. D. altus (Leconte, 1855): 366

Distribución: Campeche, Jalisco, Nayarit, Sinaloa y Veracruz. Adultos activos en julio (419), agosto (1), septiembre (566) y noviembre (1) (Short y Torres, 2006).

43. D. angustus Sharp, 1882: 78

Distribución: Campeche, Sinaloa, Tabasco y Veracruz, en altitudes de cero a $100 \mathrm{~m}$. Adultos activos en junio (1), julio (3) y agosto (2) (Short y Torres, 2006).
44. D. rudis Sharp, 1887: 765

Distribución: Campeche, Jalisco, Morelos, Sinaloa, Tabasco y Veracruz. Adultos activos en junio (627), julio (67), agosto (428), septiembre (1170) (Van Tassell, 1966; Sharp, 1887; Short y Torres, 2006).

7. Hemiosus Sharp, 1882

45. H. exilis (LeConte, 1852): 211

Distribución: Colima, Hidalgo, Jalisco, Sinaloa, y Veracruz. Adultos activos en junio (7), julio (1) y agosto (1) (Campbell et al., 2008; Short y Torres, 2006; Van Tassell, 1966).

46. H. maculatus Sharp, 1882: 85

Distribución: Baja California Sur, Colima, Guerrero, Jalisco, Morelos, Puebla, Sinaloa y Sonora. Los adultos se encuentran activos en enero (2), julio (10) y agosto (1). En Morelos se recolectó entre fanerógamas acuáticas que se desarrollan en los márgenes del río y en sustratos pedregosos a la orilla del río (Arce-Pérez, 1986; ArcePérez y Novelo-Gutiérrez, 1990; Leech, 1948b; Sharp, 1887; Short y Torres, 2006, Van Tassell, 1966).

Chaetarthriini

8. Chaetarthria Stephens, 1835

47. C. bicolor Sharp, 1882: 87

Distribución: Baja California Sur, Chiapas, Durango, Estado México, Oaxaca, San Luis Potosí, Sinaloa, Sonora y Veracruz. Especie atraída a la luz y luz negra. Adultos activos en enero (4), junio (1), julio (2), agosto (2) y diciembre (Leech, 1948b; Miller, 1974).

48. C. flava Miller, 1974: 86

Distribución: Sinaloa y Sonora. Adultos activos en mayo (1), junio (29), y agosto (42) (Miller, 1974).

49. C. hintoni Miller, 1974: 23

Distribución: Estado de México ( 7 adultos en junio), Morelos (4 adultos entre las fanerógamas acuáticas Ceratophyllum demersun que se desarrollan en las orillas del río) (Arce-Pérez, 1986; Arce-Pérez y Novelo-Gutiérrez, 1990; Miller, 1974)

50. C. magna Miller, 1974: 83

Distribución: Baja California Sur, Chihuahua y Sonora (2 adultos en agosto). Adultos activos en enero (5), agosto (17) y septiembre (1) (Miller, 1974).

51. C. major Miller, 1974: 93

Distribución: Chiapas, Oaxaca, Sinaloa y Veracruz. Adultos activos en julio (25) (Miller, 1974).

52. C. ochra Miller, 1974: 102

Distribución: Sonora. Adultos activos en febrero (1) y agosto (46) (Miller, 1974).

53. C. pallida (Leconte, 1861): 342

Distribución: Aguascalientes, Baja California, Baja California Sur, Durango, Guanajuato, Sinaloa y Sonora. Adultos activos en mayo, junio, agosto y diciembre (Miller, 1974). 
54. C. pusilla Sharp, 1882: 88

Distribución: Baja California, Baja California Sur, Chiapas, Durango, Estado de México, Morelos, Nayarit, Oaxaca, Sinaloa y Veracruz. Adultos activos en enero, junio, julio y agosto (Miller, 1974).

55. C. punctulata Sharp, 1882: 87

Distribución: Baja California, Estado de México, Sinaloa y Veracruz. Adultos activos en abril y junio (Sharp, 1882; Miller, 1974).

56. C. spangleri Miller, 1974: 82

Distribución: Chiapas, Guerrero, Oaxaca y Puebla. Adultos activos en junio (4), julio (5), agosto (1) y diciembre (1) (Miller, 1974).

57. C. veracruzensis Miller, 1974: 88

Distribución: Veracruz. Adultos activos en julio (5) y agosto (1) (Miller, 1974).

Anacaenini

9. Anacaena Thomson, 1859

58. A. signaticollis Fall, 1924: 87

Distribución: Baja California y Baja California Sur. Adultos activos en junio (1), agosto (6) y diciembre (1) (Komarek, 2005; Leech, 1945, 1948b).

59. A. solstitialis (Kirsch, 1873): 133

Distribución: Tabasco y de Toxpan sin registro estatal (Komarek, 2005; Sharp, 1882).

60. A. suturalis (Leconte, 1866): 366

Distribución: Baja California Sur, Distrito Federal, Guerrero, Michoacán, Morelos y Veracruz. Adultos activos en abril (1), julio (1), agosto (1) y noviembre (1), en Morelos están presentes casi todo el año; enero (5), febrero (184), marzo (50), junio (19), julio (45), agosto (330), octubre (775), noviembre (99), diciembre (46) entre las fanerógamas acuáticas, Ceratophyllum demersum y Ludwigia repens que se desarrollan a la orilla del río $\mathrm{y}$, en estanques temporales con gramíneas emergentes (ArcePérez, 1986; Arce-Pérez y Novelo-Gutiérrez, 1990; Leech, 1948b; Komarek, 2005; Santiago-Fragoso y MejoradaGómez, 1999).

10. Notionotus Spangler, 1972

61. N. mexicanus Perkins, 1980: 306

Distribución: Oaxaca (Hansen, 1999).

11. Paracymus Thomson, 1867

62. P. armatus (Sharp, 1882): 64

Distribución: Hidalgo y Sinaloa (Campbell et al., 2008; Sharp, 1887).

63. P. confusus Wooldridge, 1966: 719

Distribución: Durango, Morelos y Sinaloa. En Morelos adultos activos casi todo el año; enero (231), febrero (206), marzo (1), mayo (6), junio (107), julio (69), agosto (37), octubre (239) y diciembre (39), entre las fanerógamas acuáticas, Ceratophyllum demersum y Ludwigia repens que se desarrollan a la orilla del río y en estanques tem- porales con gramíneas emergentes (Arce-Pérez, 1986; Arce-Pérez y Novelo-Gutiérrez, 1990; Wooldridge, 1966). 64. P. corrinae Wooldridge, 1969: 418

Distribución: Veracruz. Adultos activos en julio (1) y agosto (7) (Wooldridge, 1969).

65. P. elegans (Fall, 1901): 218

Distribución: Baja California. Adultos activos en junio (3) (Leech, 1948b).

66. P. ellipsis (Fall, 1910): 99

Distribución: norte y noroeste de México (Wooldridge, 1969).

67. P. indigens Wooldridge, 1969: 419

Distribución: Campeche, Guerrero, Oaxaca, Tabasco, Veracruz. Adultos activos en julio (51) y agosto (9) (Wooldridge, 1969).

68. P. leechi Wooldridge, 1969: 413

Distribución: Durango, Hidalgo, Jalisco, Michoacán, Puebla, San Luis Potosí, Tlaxcala y Zacatecas. Adultos activos en junio (34), julio (69), agosto (6), octubre (8), noviembre

(4) y diciembre (6) (Campbell et al., 2008; Wooldridge, 1969).

69. P. mexicanus Wooldridge, 1969: 415

Distribución: Distrito Federal, Jalisco, Michoacán, Puebla. Adultos activos en abril-mayo (2), julio (11) y diciembre (1) (Santiago-Fragoso y Mejorada-Gómez, 1999; Wooldridge, 1969).

70. P. nanus (Fall, 1910): 99

Distribución: Costas del golfo de México (Wooldridge, 1969).

71. P. regularis Wooldridge, 1969: 417

Distribución: Chiapas, Michoacán, Oaxaca, Puebla, Veracruz. Adultos activos en enero (23), julio (61), agosto (9) y diciembre (12) (Wooldridge, 1969).

72. P. secretus Wooldridge, 1973: 436

Distribución: Durango. Adultos activos en junio (14) (Wooldridge, 1973).

73. P. securus Wooldridge, 1975: 81

Distribución: Baja California (Hansen, 1999).

74. P. subcupreus (Say, 1825): 189

Distribución: Guanajuato, Veracruz, Yucatán (Darlington, 1936; Sharp, 1882)

75. P. toleratus Wooldridge, 1973: 434

Distribución: Oaxaca. Adultos activos en mayo (9) (Wooldridge, 1973).

Oocyclini

12. Laccobius Erichson 1837

76. L. borealis Cheary, 1971: 110

Distribución: Baja California (Gentili, 1986a).

77. L. californicus Orchymont, 1942: 17

Distribución: Baja California (Gentili, 1986b).

78. L. carri Orchymont, 1942: 12

Distribución: Baja California. Adultos activos en julio (1) 
(Gentili, 1986b).

79. L. ellipticus LeConte, 1855: 363

Distribución: Baja California, Baja California Sur, Guanajuato y Puebla. En la península de Baja California, adultos activos en marzo (1), junio (2); julio (18) y agosto (1) (Leech, 1948b; Sharp, 1882; Gentili, 1986b).

80. L. hardyi Cheary, 1971: 105

Distribución: Durango, Sonora. Adultos activos en julio (1) y agosto (148) (Gentili, 1986b).

81. L. hintoni Orchymont, 1942: 8

Distribución: Sinaloa. Adultos activos en mayo (3); junio (32) y agosto (1) (Gentili, 1986b; van Tassell, 1966).

82. L. mexicanus Orchymont, 1942: 17

Distribución: Baja California, Baja California Sur, Coahuila, Chihuahua, Distrito Federal, Durango, Guanajuato, Hidalgo, Estado de México, Oaxaca, Puebla, San Luis Potosí, Sonora, Tabasco, Tlaxcala, Veracruz y Zacatecas. Adultos activos en febrero (13), marzo (85), junio (9), julio (40), agosto (9) y octubre (2) (Campbell et al., 2008; Gentili, 1986b; Sharp, 1882).

13. Oocyclus Sharp, 1882

83. E. brevicornis Sharp, 1882: 63

Distribución: Nayarit, Oaxaca y Veracruz. Adultos activos en marzo (75), julio (30) y septiembre (5) (Short y Perkins, 2004).

84. E. grandis Short y Perkins, 2004: 11

Distribución: Jalisco, Nayarit, Oaxaca y Veracruz. Adultos activos en marzo (1), julio (97), septiembre (42) (Short y Perkins, 2004).

85. E. vestitus Sharp, 1882: 62

Distribución: Durango, Jalisco, Veracruz. Adultos activos en julio (4) (Sharp 1882; Short y Perkins, 2004).

Hydrophilini

14. Cymbiodyta Bedel, 1881

86. C. brevicollis (Sharp, 1882): 71

Distribución: Durango, Jalisco, Michoacán, Oaxaca y Puebla. Adultos activos en noviembre (1) y diciembre (2) (Sharp, 1882; Smetana, 1974).

87. C. brevipalpis brevipalpis Smetana, 1974: 16

Distribución: Jalisco. Adultos activos en diciembre (18) (Smetana, 1974).

88. C. brevipalpis pygmaea Smetana, 1974: 16

Distribución: Morelos. Los ejemplares fueron recolectados al cernir la hojarasca de ambientes húmedos cerca de la orilla de cuerpos de agua, por lo que se considera una especie de hábitos semiacuáticos.Recolectada junto con ejemplares de C. polita y C. campbelli (1 adulto en marzo, 1 en abril y 6 en diciembre), Oaxaca (26 adultos en mayo) (Navarrete-Heredia, 1992; Smetana, 1974).

89. C. campbelli Smetana, 1974: 18

Distribución: Oaxaca (6 adultos en mayo). Material recolectado al cernir hojarasca cercana de los bordes de pequeños arroyos junto con $C$. polita y $C$. brevipalpis pygmaea (Smetana, 1974).

90. C. dorsalis (Motschulsky, 1859): 177

Distribución: Baja California, Baja California Sur, Chihuahua. Esta especie fue recolectada en junio (7) y julio (1) en pantanos, arroyos pedregosos, estanques y manantiales (Leech, 1945, 1948b; Smetana, 1974).

91. C. fraterculus (Sharp, 1882): 71

Distribución: Chihuahua (7 adultos en julio), Guanajuato, Puebla, Sonora (Sharp, 1882, 1887; Smetana, 1974).

92. C. howdeni Smetana, 1974: 20

Distribución: Durango (2 adultos en junio) (Smetana, 1974).

93. C. polita (Sharp, 1882): 72

Distribución: Chiapas, Morelos (1 adulto en septiembre) y Oaxaca. Adultos recolectados en arroyos pequeños; en Oaxaca recolectados al cernir hojarasca cercana a los bordes de pequeños arroyos junto con $C$. campbelli y $C$. brevipalpis pygmaea (Smetana, 1974).

94. C. pusilla Smetana, 1974: 18

Distribución: Durango (2 adultos en diciembre) (Smetana, 1974).

15. Enochrus Thomson, 1859

95. E. aequalis (Sharp, 1882): 68

Distribución: Guanajuato, Oaxaca, Veracruz (Sharp, 1882).

96. E. californicus (Horn, 1890): 248

Distribución: Baja California Sur (1 adulto en junio) (Leech, 1948b).

97. E. carinatus carinatus (LeConte, 1855): 370

Distribución: Baja California, Baja California Sur (10 adultos en julio) y Hidalgo (Campbell et al., 2008; Leech, 1948a).

98. E. carinatus fucatus Horn, 1873: 127

Distribución: Chihuahua, Guanajuato, Sinaloa y Sonora (Gundersen, 1977, 1978).

99. E. cristatus (LeConte, 1855): 370

Distribución: Chihuahua, Guanajuato, Hidalgo, Jalisco, Michoacán, Nuevo León, Oaxaca, Puebla, Querétaro, San Luis Potosí, Tamaulipas, Veracruz y Zacatecas (Campbell et al., 2008; Sharp, 1882; Gundersen, 1978).

100. E. debilis (Sharp, 1882): 69

Distribución: Chiapas, Hidalgo, Nayarit, Oaxaca, Querétaro, Tamaulipas, Veracruz y Yucatán (Gundersen, 1978).

101. E. fimbriatus (Melsheimer, 1844): 101

Distribución: Sinaloa y Sonora (Gundersen, 1978).

102. E. metacarina Short, 2005: 203

Distribución Veracruz (1 adulto en junio y 1 en agosto) (Short, 2005b; Short y Hebauer, 2006).

103. E. mexicanus (Sharp, 1882): 67

Distribución: Colima, Distrito Federal, Jalisco, Estado de México, Hidalgo, Morelos, Oaxaca, Puebla y Veracruz (Campbell et al., 2008; Santiago-Fragoso y Mejorada- 
Gómez, 1999; Sharp, 1882; Gundersen, 1978).

104. E. obscurus (Sharp, 1882): 69

Distribución: Oaxaca y Yucatán (Sharp, 1882; Darlington, 1936).

105. E. ochraceus (Melsheimer, 1844): 101

Distribución: Campeche, Distrito Federal, Guanajuato, Jalisco, Michoacán, Sinaloa, Sonora, Tabasco y Veracruz (Santiago-Fragoso y Mejorada-Gómez, 1999; Gundersen, 1978).

106. E. plicatus Short, 2005: 2

Distribución: Jalisco (2 adultos en diciembre) (Short, 2005a; Short y Hebauer, 2006).

107. E. pseudochraceus Gundersen, 1977: 256

Distribución: Colima, Chiapas, Jalisco, Nayarit, Oaxaca, Sinaloa, Tabasco, Tamaulipas y Veracruz (Gundersen, 1978).

108. E. pygmaeus pygmaeus (Fabricius, 1792): 186

Distribución: Baja California Sur (2 adultos en julio), Hidalgo, Jalisco, Morelos, Sinaloa, Sonora y Veracruz. En Morelos se recolectaron en enero (28); febrero (2), marzo (12), abril (6), octubre (10) y noviembre (2); a la orilla del río, en abundante vegetación y escasa profundidad con sustrato pedregoso y fangoso (Arce-Pérez y NoveloGutiérrez, 1990; Campbell et al., 2008; Leech, 1948b; Gundersen, 1978).

109. E. pygmaeus pectoralis LeConte, 1855: 370

Distribución: Baja California, Baja California Sur (190 adultos en julio), Colima, Chiapas, Durango, Guanajuato, Guerrero, Jalisco, Estado de México, Hidalgo, Morelos, Oaxaca, San Luis Potosí, Sinaloa, Sonora y Veracruz. En Morelos se encuentra adultos casi todo el año; febrero (3), marzo (59), mayo (207), junio (476), julio (17), octubre (33) y diciembre (5) en las orillas del río entre fanerógamas acuáticas Ceratophyllum demersum y Ludwigia repens, algunos en estanques temporales (Arce-Pérez, 1986; Arce-Pérez y Novelo-Gutiérrez, 1990; Leech, 1948b; Gundersen, 1978).

110. E. sharpi Gundersen, 1977: 262

Distribución: Aguascalientes, Chiapas, Jalisco, Estado de México, Michoacán, Morelos (Tetelcingo-Anenecuilco), Nayarit; Puebla, Sinaloa, Tabasco y Veracruz (Gundersen, 1978; Santiago-Fragoso y Sandoval-Manrique, 2001).

111. E. spangleri Santiago- Fragoso y Mejorada-Gómez, 1995: 36

Distribución: Chiapas (4 adultos en mayo) (Santiago-Fragosos y Mejorada-Gómez, 1995).

16. Helobata Bergroth, 1888

112. H. larvalis (Horn, 1873): 137

Distribución: Sonora (Fernández y Bachmann, 1987; Sharp, 1882).

17. Helochares Mulsant, 1844a

113. H. maculicollis Mulsant, 1844b: 379
Distribución: "Baja California", Morelos, Veracruz y Yucatán. En Morelos se encuentra la mayor parte del año; enero (3), marzo (20), mayo (28), junio (35), julio (111), agosto (6), octubre (24), noviembre (5) y diciembre (4), en estanques temporales poco profundos con gramíneas emergentes y fanerógamas acuáticas Ceratophyllum demersum y Ludwigia repens (Arce-Pérez, 1986; ArcePérez y Novelo-Gutiérrez, 1990; Darlington, 1936; Leech, 1945; Sharp, 1882).

114. H. mundus (Sharp, 1882): 73

Distribución: Campeche, Jalisco y Oaxaca. Adultos activos en julio (28) (Sharp, 1882; Short, 2005b).

115. H. normatus (LeConte, 1861): 341

Distribución: Aguascalientes, Baja California Sur, Colima, Chihuahua, Guerrero, Hidalgo, Morelos y Sinaloa. Adultos activos en junio (21), agosto (1), en Morelos presentes casi todo el año; noviembre (7), diciembre (1), enero (13), febrero (3), marzo (11), abril (9), mayo (1) (Campbell et al., 2008; Leech, 1945, 1948b; Santiago-Fragoso y VázquezNavarrete, 1989; Sharp, 1882, 1887; Short, 2005b).

116. H. sallaei Sharp, 1882: 75

Distribución: Veracruz (Sharp, 1882).

117. H. optatus (Sharp, 1882):72

Distribución: Tamaulipas y Veracruz. Adultos activos en julio (6) y agosto (3) a la luz negra (Short, 2005b).

18. Hydrobiomorpha Blackburn, 1888

118. H. casta (Say, 1835): 170

Distribución: Veracruz (Bachmann, 1988; Sharp, 1882, 1887).

19. Hydrochara Berthold, 1827

119. H. leechi Smetana, 1980: 38

Distribución: Chihuahua. Adultos en junio (6) (Smetana, 1980).

120. H. lineata (LeConte, 1855): 369

Distribución: Baja California. Adultos activos en marzo (1), abril (11), mayo (1), junio (3) y agosto (2) (Leech, 1948b; Smetana, 1980).

20. Hydrophilus Geoffroy, 1762

121. H. ensifer Brullé, 1837: 52

Distribución: Veracruz, Yucatán (Darlington, 1936; Sharp, 1882).

122. H. insularis Castelnau, 1840: 50

Distribución: Baja California Sur, Morelos, Puebla, Sinaloa, Veracruz y Yucatán. En Morelos se encontraron adultos en febrero (2), marzo (2), junio (2), Julio (4) $\mathrm{y}$ agosto (2), en estanques temporales profundos formados por el remanso del río, con fanerógamas acuáticas Eichornia crassipes, escasos en estanques temporales con gramíneas emergentes (Arce-Pérez, 1986; Arce-Pérez y Novelo-Gutiérrez, 1990; Darlington, 1936; Leech, 1948b; Regimbart, 1901; Sharp, 1882).

123. H. pollens Sharp, 1887: 762 
Distribución: Veracruz (Sharp, 1887).

124. H. smaragdinus Brullé, 1837: 54

Distribución: Veracruz (Sharp, 1887).

125. H. triangularis Say, 1823: 201

Distribución: Morelos, Oaxaca, San Luis Potosí y Veracruz. En Morelos se encontraron adultos en febrero (10), marzo (6), junio (1) y octubre (1), estanques temporales profundos formados por remansos del río, con fanerógamas acuáticas Eichornia crassipes, escasos en estanques temporales con gramíneas emergentes (Arce-Pérez, 1986; Arce-Pérez y Novelo-Gutiérrez, 1990; Regimbart, 1901; Sharp, 1882).

\section{Tropisternus Solier, 1834}

126. T. afinis Motschulsky, 1859: 175

Distribución: Baja California, Baja California Sur, Chiapas, Chihuahua, Coahuila, Colima, Distrito Federal, Durango, Guanajuato, Hidalgo, Jalisco, Estado de México, Michoacán, Morelos, Nayarit; Nuevo León, Oaxaca, Puebla, Querétaro, San Luis Potosí, Sonora, Tamaulipas y Zacatecas. En la península de Baja California Adultos activos en (junio (11), julio (21), agosto (3). En Morelos los adultos están presentes casi todo el año; enero (11), febrero (36), marzo (33), abril (20), mayo (54), junio (54), agosto (1); septiembre (1), octubre (20), noviembre (8), diciembre (20) en estanques temporales con gramíneas emergentes y fanerógamas acuáticas Ceratophyllum demersum, Ludwigia repens; también entre las fanerógamas acuáticas que se desarrollan en los márgenes del río (Arce-Pérez, 1986; Arce-Pérez y Novelo-Gutiérrez, 1990; Arce-Pérez y Novelo-Gutiérrez, 1991; Campbell et al., 2008; Leech, 1948b; Santiago-Fragoso y Vázquez-Navarrete, 1989; Sharp, 1882; Spangler, 1960).

127. T. apicipalpis Chevrolat, 1834: 44

Distribución: Baja California Sur (4 adultos en julio), Chiapas, Durango, Jalisco, Estado de México, Michoacán, Morelos, Nayarit, Querétaro, San Luis Potosí, Veracruz y Yucatán. En Morelos se recolectaron en febrero (11), marzo (17), agosto (2), septiembre (2) y octubre (1); en estanques temporales profundos con fanerógamas acuáticas Ceratophyllum demersum, Ludwigia repens, y algunos en estanques con gramíneas emergentes a la orilla del río (Arce Pérez, 1986; Arce-Pérez y Novelo-Gutiérrez, 1990; Arce-Pérez y Novelo-Gutiérrez, 1991; Darlington, 1936; Leech, 1948b; Sharp, 1882; Spangler, 1960).

128. T. baeri Orchymont, 1921: 362

Distribución: Campeche, Nayarit, San Luis Potosí, Sinaloa, Tabasco y Veracruz. Adultos activos en febrero (1), junio (3), septiembre (3) y febrero (1) (Spangler, 1960).

129. T. brevicollis Sharp, 1882: 56

Distribución: Chiapas, Durango, Guerrero, Jalisco, Michoacán, Nayarit, Oaxaca y Sonora. Adultos activos en abril (1), julio (2), septiembre (2), diciembre (1) (Spangler,
1960; Sharp, 1882).

130. T. californicus (LeConte, 1855): 367

Distribución: Baja California, Baja California Sur. Adultos activos en junio (3) (Leech, 1948b; Spangler, 1960).

131. T. columbianus Brown, 1931:117

Distribución: Baja California, Distrito Federal, Estado de México y Sonora. Los adultos activos en junio (1) y octubre (1) (Santiago-Fragoso y Mejorada-Gómez 1999; Spangler, 1960).

132. T. collaris mexicanus Castelnau, 1840: 54

Distribución: Campeche, Coahuila, Colima, Chiapas, Guerrero, Jalisco, Estado de México, Michoacán, Morelos, Nayarit, Nuevo León, Oaxaca, San Luis Potosí, Sinaloa, Sonora, Tabasco, Tamaulipas y Veracruz. En Morelos son abundantes casi todo el año, en enero (225), febrero (6), marzo (83), abril (20), mayo (197), junio (1777), julio (202), agosto (104), septiembre (431), octubre (48), noviembre (207), diciembre (198) en estanques temporales con gramíneas emergentes y fanerógamas acuáticas Ceratophyllum demersum, Ludwigia repens; también entre las fanerógamas acuáticas que se desarrollan en los márgenes del río (Arce-Pérez, 1986; Arce-Pérez y Novelo-Gutiérrez, 1990; Santiago-Fragoso y Vázquez-Navarrete, 1989; Santiago-Fragoso y Sandoval-Manrique, 2001; Sharp, 1882; Spangler 1960).

133. T. chalybeus Castelnau, 1840: 53

Distribución: Chiapas, Colima, Oaxaca, Veracruz y Yucatán. Adultos activos en junio (3), julio (3) y diciembre (6) (Darlington, 1936; Spangler, 1960).

134. T. fuscitarsis Sharp, 1882: 58

Distribución: Chiapas, Colima, Distrito Federal, Jalisco, Estado de México, Michoacán, Morelos, Nayarit, Oaxaca, Puebla, Querétaro, San Luis Potosí, Sonora y Veracruz. Adultos activos en enero (1), junio (8), julio (1), agosto (10), septiembre (14), noviembre (7) y diciembre (38) (Sharp, 1882; Spangler, 1960).

135. T. lateralis limbalis (LeConte, 1855): 367

Distribución: Colima, Sinaloa y Sonora. Adultos activos en enero (15), febrero (1), abril (3), junio (39), julio (22), agosto (26) y septiembre (4) (Sharp, 1887; Spangler, 1960). 136. T. lateralis nimbatus (Say, 1823): 203

Distribución: Baja California, Baja California Sur, Chiapas, Coahuila, Colima, Distrito Federal, Durango, Guanajuato, Hidalgo, Jalisco, Estado de México, Michoacán, Morelos, Nuevo León, Oaxaca, Puebla, Querétaro, San Luis Potosí, Sinaloa, Sonora y Veracruz. En Morelos son comunes en enero (10), febrero (28), marzo (39), abril (27), mayo (9), junio (61), julio (26), septiembre (3), octubre (19) y diciembre (53), en márgenes someros del río provistos de fanerógamas acuáticas Ceratophyllum demersum y Ludwigia repens y escasos en estanques temporales con gramíneas emergentes (Arce-Pérez, 1986; Arce-Pérez y 
Novelo-Gutiérrez, 1990; Arce-Pérez y Novelo-Gutiérrez, 1991; Campbell et al., 2008; Leech, 1948b; SantiagoFragoso y Mejorada-Gómez, 1999; Santiago-Fragoso y Sandoval-Manrique, 2001; Sharp, 1882; Spangler, 1960). 137. T. mergus Say 1835: 171

Distribución: Baja California, Colima, Durango, Guerrero, Jalisco, Michoacán, Morelos, Nayarit, Oaxaca, Puebla, Sonora y Veracruz. Adultos activos casi todo el año; enero (39), febrero (45), marzo (60), abril (15), mayo (5), junio (18), julio (14), septiembre (65), octubre (5), noviembre (8), diciembre (22) (Leech, 1948b, Santiago-Fragoso y Vázquez-Navarrete, 1989; Sharp, 1882; Spangler, 1960).

138. T. niger Orchymont, 1938: 438

Distribución: Nayarit (1 adulto en noviembre) y Sinaloa. Adultos activos en noviembre (2) (Spangler, 1960).

139. T. obscurus Sharp.1882: 60

Distribución: Baja California Sur, San Luis Potosí, Sinaloa y Veracruz. Adultos activos en junio (4) y julio (2) (Leech, 1948b; Sharp, 1887; Spangler, 1960).

140. T. oculatus Sharp, 1882: 58

Distribución: Durango, Sinaloa y Sonora (1 adulto en julio) (Sharp, 1882; Spangler, 1960).

141. T. ovalis Castelnau, 1840: 54

Distribución: Nayarit y Veracruz (Sharp, 1882; Spangler, 1960).

142. T. quadristriatus (Horn, 1871): 331

Distribución: Baja California, Nayarit y Yucatán (3 adultos en agosto) (Spangler, 1960).

143. T. sublaevis (LeConte. 1855): 368

Distribución: Aguascalientes, Chihuahua, Durango, Oaxaca, Sonora, Veracruz y Yucatán (Arce-Pérez y Novelo-Gutiérrez, 1991; Darlington, 1936; Sharp, 1882, 1887; Spangler, 1960).

144. T. tinctus Sharp, 1882: 59

Distribución: Distrito Federal, Estado de México, Oaxaca, Puebla, Querétaro, San Luis Potosí y Veracruz. Adultos activos en enero (1), abril (2), mayo (2), junio (7) julio (1) agosto (9), septiembre (2) y diciembre (10) (SantiagoFragoso y Mejorada-Gómez, 1999; Sharp, 1882, Spangler, 1960).

145. T. salsamentus Fall, 1901: 204

Distribución: Baja California (Leech, 1948b).

Sphaeridiinae

Coelostomatini

22. Dactylosternum Wollaston, 1854

146. D. abdominale Fabricius, 1792: 79

Distribución: Baja California y Tabasco (Sharp, 1887).

147. D. cacti (LeConte, 1855): 373

Distribución: Baja California y Baja California Sur. Adultos activos en junio (14), recolectados en cardón, cactus y Pachycerus podridos (Leech, 1948b).

148. D. dispar (Sharp, 1882): 95
Distribución: Guanajuato, Hidalgo y Tabasco (Sharp, 1882, 1887).

149. D. subdepressum (Castelnau, 1840): 58

Distribución: Oaxaca y Veracruz (Sharp, 1882).

23. Phaenonotum Sharp, 1882

150. P. apicale Sharp, 1882: 98

Distribución: Morelos (Sharp, 1887).

151. P. exstriatum (Say, 1835): 171

Distribución: Baja California, Baja California Sur (6 adultos en julio), Morelos, Oaxaca, Sonora, Tabasco y Veracruz (Leech, 1948b; Sharp, 1882, 1887). Se corrobora su presencia en Veracruz, municipio de Catemaco, Ejido Adolfo López Mateos (182 26’ 4.925” N y 94 57'35.185" O), pastizal 1/5-XII-2003, Martín de los Santos Bailón col. $1 \hat{0}, 2$.

152. P. globulosum (Mulsant, 1844a): 134

Distribución: Veracruz (Sharp, 1882).

153. P. rotundulum Sharp, 1882: 100

Distribución: Veracruz (Sharp, 1882).

Megasternini

24. Agna Smetana, 1978

154. A. capillata (LeConte, 1855): 374

Distribución: Baja California (Leech, 1948b).

155. A. rufescens (Horn, 1895): 233

Distribución: Baja California Sur (Blackwelder, 1931; Hansen, 1999; Leech, 1948b).

25. Cercyon Leach, 1817

156. C. aequatus Knisch, 1924: 131

Distribución: Veracruz (Sharp, 1887).

157. C. fimbriatus Mannerheim, 1852: 344

Distribución: Baja California, Baja California Sur (49 adultos en agosto) (Leech, 1948b).

158. C. integer Sharp, 1882: 106

Distribución: Veracruz (Sharp, 1882).

159. C. luniger Mannerheim, 1853: 168

Distribución: Baja California y Baja California Sur (2 adultos en agosto) (Leech, 1948b).

160. C. mellipes (Say, 1835): 172

Distribución: México (necesita confirmación, Hansen, 1999).

161. C. mexicanus Sharp, 1882: 104

Distribución: Veracruz (Sharp, 1882).

162. C. politus Sharp, 1882: 105

Distribución: Veracruz (Sharp, 1882).

163. C. praetextatus (Say, 1825): 190

Distribución: Baja California, Distrito Federal, Tabasco

y Veracruz (Santiago-Fragoso, 1999 y Mejorada-Gómez; Sharp, 1882, 1887; Smetana, 1978).

164. C. spathifer Smetana, 1978: 149

Distribución: Baja California (Smetana, 1978).

165. C. variegatus Sharp, 1882: 107

Distribución: Oaxaca y Veracruz (Sharp, 1882). Se corro- 
bora su presencia en Veracruz, municipio de Catemaco, ejido Adolfo López Mateos, en trampa de pozo, mayo

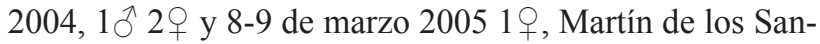
tos Bailón col.

26. Cryptopleurum Mulsant, 1844a

166. C. impressum Sharp, 1882: 115

Distribución: Baja California (5 adultos en junio), Estado de México, Morelos, Oaxaca y Veracruz, (Hinton y Ancona, 1934; Leech, 1948b; Sharp, 1882).

27. Cycrillum Knisch., 1921

167. C. strigicolle (Sharp, 1882): 113

Distribución: Chiapas, Oaxaca y Veracruz. Adultos activos en mayo (16), junio (1) y julio (1) (Sharp, 1882; Smetana, 1984).

28. Deltostethus Sharp, 1882

168. D. columbiensis (Hatch, 1965): 45

Distribución: Durango y Estado de México. Adultos activos en junio (1) y julio (10) (Smetana, 1978, 1985b).

169. D. scitulus Spangler y Huacuja, 1982: 1

Distribución: Hidalgo. Adultos activos en marzo (1), mayo (2), junio (2), julio (7) y octubre (11) (Spangler y Huacuja, 1982).

29. Megasternum Mulsant, $1844 \mathrm{a}$

170. M. posticatum (Mannerheim, 1852): 344

Distribución: Baja California (Leech, 1948a).

30. Motonerus Hansen, 1989

171. M. obscurus Hansen, 1989: 257

Distribución: Chiapas, Hidalgo y Oaxaca. Adultos activos en mayo (1), julio (11), septiembre (1) (Fikácek y Short, 2006).

31. Oosternum Sharp, 1882

172. O. costatum (LeConte, 1855): 374

Distribución: Estado de México, Morelos y Puebla (Hinton y Ancona 1934; Sharp, 1882).

173. O. attacomis Spangler, 1962: 98

Distribución: Morelos. Adultos activos en enero (3), marzo (1), abril (1), julio (155), septiembre (3), octubre (2) (Márquez-Luna y Navarrete-Heredia, 1995). Oosternum attacomis Spangler, se registra por primera vez para Veracruz en el municipio de Soteapan, ejido San Fernando $\left(18^{\circ}\right.$ 19’6.334" N y 94 52'53.474"O), pastizal, del 19-22 de enero 2004, Martín de los Santos Bailón col. 1 q .

32. Pelosoma Mulsant, 1844a

174. P. cercyonoides Sharp, 1882: 111

Distribución: "México" (necesita confirmación, Smetana, 1984).

175. P. lafertei Mulsant, 1844a: 134

Distribución: Veracruz (Sharp, 1882).

176. P. mesosternalum Hinton y Ancona, 1934: 246

Distribución: Estado de México (Hinton y Ancona, 1934).

177. P. ovulm Sharp, 1887: 772

Distribución: Estado de México (Sharp, 1887).
178. P. pecki Smetana, 1984:561

Distribución: Oaxaca. Adultos activos en mayo (6) (Smetana, 1984).

179. P. prosternale Sharp, 1882: 110

Distribución: Guanajuato y Coahuila (Sharp, 1882; Smetana, 1984).

180. P. sordidum Sharp, 1882: 109

Distribución: Veracruz y Tabasco (Sharp, 1882).

Omicrini

33. Omicrus Sharp, 1879

181. O. globulus (Sharp, 1882): 102

Distribución: Veracruz (Sharp, 1882).

182. O. intermedius Smetana, 1975: 164

Distribución: "sur de México" (Smetana, 1984).

183. O. laevis (Sharp, 1887): 770

Distribución: Baja California (Smetana, 1975).

184. O. seriatus Smetana, 1975: 163

Distribución: Chiapas (Hansen, 1999).

34. Heteryon Sharp, 1882

185. H. depressus Sharp, 1882: 103

Distribución: Veracruz (Sharp, 1882).

35. Aculomicrus Smetana, 1990

186. A. minimus Smetana, 1975: 179

Distribución: Chiapas (Hansen, 1999).

Sphaeridiini

36. Sphaeridium Fabricius, 1775

187. S. scarabaeoides (Linneo, 1758): 256

Distribución: Distrito Federal (Hendrich, 1975; Smetana, 1978). Ahora se registra por primera vez para el estado de Veracruz en el municipio de Las Vigas de Ramírez, Manzanillo (19³8'18' N, 97 07'75' O), $2466 \mathrm{~m}$ de altitud, Rancho Los Castaños, en excremento de caballo, 31-VII-2009, R. Arce col. 3 adultos.

\section{Discusión}

No obstante que el tamaño de los adultos, su abundancia y riqueza, hábitos acuáticos, riparios y terrestres, así como su importancia biológica, médica y económica los hacen un excelente grupo de estudio, ningún trabajo se conoce que trate a la superfamilia en México. Sin embargo, el reciente crecimiento en el conocimiento y valor de la biodiversidad de los ecosistemas acuáticos, han incrementado el estudio de estos organismos, y con ayuda de la información aquí expuesta será posible reconocer y describir muchas de las especies de hidrofiloideos de México, así como iniciar estudios sistemáticos, biológicos y de distribución. El registro de 27 géneros y 129 especies que se tenía para México (Arce-Pérez, 1995) aumentó a 36 géneros, 181 especies y 6 subespecies, lo que repre- 
senta un incremento del $25 \%$ y $28 \%$ respectivamente; esta cifra no revela una diversidad baja sino más bien la falta de recolecciones y estudios faunísticos en todos los estados y ambientes acuáticos del país, que a su vez indica la necesidad de conservar representantes de todos los tipos de vegetación en las áreas naturales protegidas del territorio, y con esto la permanencia de los diferentes cuerpos de agua donde se desarrollan éste y otros grupos de invertebrados dulceacuícolas. Por último, es interesante destacar la presencia de Cercyon variegatus, Phaenonotum extriatum y Oosternum atacomis en monolitos de tierra en la región de Los Tuxtlas, Veracruz, lo cual pone en evidencia su capacidad para vivir en suelos húmedos, y su captura por trampas de pozo indica que posiblemente estaban en vuelo de dispersión entre diciembre y mayo.

\section{Agradecimientos}

A Isabelle Barois (INECOL, Xalapa) por su apoyo para la realización de este estudio, resultado parcial del proyecto "Conservation and sustainable management of below-grown Bio-Diversity-Mexico" (GF/2715-02, GEF/ BGBD), y a Martín de los Santos Bailón, por la recolección y separación de muestras de coleópteros obtenidas durante dicha investigación. A Andrew Edward Z. Short, de la Univesidad de Kansas, por la revisión del manuscrito.

\section{Literatura citada}

Anderson, J.M. y J.S. Ingram. 1993. Tropical soil biology and fertility: A handbook of methods. CAB International, Oxford. $221 \mathrm{p}$.

Arce-Pérez, R. 1986. Contribución al conocimiento de los coleópteros acuáticos del río Amacuzac, en la región de Vicente Aranda, Morelos, México. Tesis, Facultad de Ciencias, Universidad Nacional Autónoma de México, México, D.F. 130 p.

Arce-Pérez, R. 1995. Lista preliminar de coleópteros acuáticos del estado de Morelos, México. Acta Zoológica Mexicana (n.s.) 65:43-53.

Arce-Pérez, R. y R. Novelo-Gutiérrez. 1990. Contribución al conocimiento de los coleópteros acuáticos del río Amacuzac, Morelos, México. Folia Entomológica Mexicana 78:29-47.

Arce-Pérez, R. y R. Novelo-Gutiérrez. 1991. Coleópteros acuáticos de la Reserva de la Biosfera La Michilía Durango, México. Folia Entomológica Mexicana 81:341-344.

Archangelsky, M. 1998. Phylogeny of Hydrophiloidea (Coleoptera: Staphyliniformia) using characters from adult and preimaginal stages. Systematic Entomology 23:9-24.
Arnett, R. A. 1972. The beetles of the United States: a manual for identification. The American Entomological Institute, Ann Arbor, Michigan. $1112 \mathrm{p}$.

Bachmann, A. O. 1988. Las especies americanas de Hydrobiomorpha (Coleoptera: Hydrophilidae). Opera Lilloana 36:1-63.

Bedel, L. 1881. Faune des Coléoptères du bassin de la Seine vol. 1. Annales de la Société Entomologique de France 24:1-360.

Bergroth, E. 1888. Fáhraea nov. gen. Hydrophilidarum. Deutsche Entomologische Zeitschrift 32:221.

Berthold, A. A. 1827. Latreille's Natürliche Familien des Thierreichs (German Translation of Latreille: Familles Naturelles...1825). Gr. H. S. priv. Landes-IndustrieComptoirs, Weimar. 604 p.

Beutel, R. G. 1994. Phylogenetic analysis of Hydrophiloidea based on characters the head of adults and larvae (Coleoptera: Staphyliniformia). Koleopterologische Rundschau, 64:103-131.

Blackburn, T. 1888. Notes on Australian Coleoptera with descriptions of new species. Proceedings of the Linnean Society of New South Wales 3 (1889):805-875.

Blackwelder, R. E. 1931. The Sphaeridiinae of the Pacific Coast. Pan-Pacific Entomologist 8:19-32.

Blackwelder, R. E. 1944. Checklist of the Coleopterous insects of Mexico, Central America, the West Indies, and South America, part I. Bulletin of the United States National Museum 185:1-188.

Brullé, A. 1837. Famille des Hydrophiliens. In Voyage dans 1' Amérique méridionale, vol. 6, part. 2. Insectes (1836-1847) A. d'Orbigny, P. Bertrand, Paris, Estrasburgo. p. 52-56.

Campbell, B. W., R. Arce-Pérez y Gómez-Anaya, J. A. 2008. Taxonomic distinctness and aquatic Coleoptera: comparing a perennial and intermittent stream with differing geomorphologies in Hidalgo, Mexico. Aquatic Ecology 42:103-113.

Castelnau, F. L. Laporte de.1840. Histoire naturelle des animaux articués. Histoire naturelle des Insectes Coléoptères, vol. 2, (Nécrophages-Triméres). P. Duménil, Paris. 565 p.

Cortés-Aguilar, J., J. L. Navarrete-Heredia, V. H. Gómez-Flores, M. Vásquez-Bolaños, E. López-Contreras, G. A. QuirozRocha y G. Labrador Chávez. 2004. Lepiceridae (Coleoptera: Myxophaga) y Georissidae (Coleoptera: Polyphaga): dos familias de Coleoptera poco conocidas. Entomología Mexicana 3:804-809.

Chandler, D. S. 1973. New records for Georyssidae and Cyatoceridae (Coleoptera) in Mexico. Pan-Pacific Entomology 49:288.

Cheary, B. S. 1971. The biology, ecology and systematics of the genus Laccobius (Laccobius) (Coleoptera: Hydrophilidae) of the New World. University of California, Riverside. 178 p.

Chevrolat, L. A. A. 1834. Coléoptères du Mexique, fasc. 3. G. Silbermann, Strasbourg. 48 p. 
Darlington, P. J. 1936. XVI. Aquatic Coleoptera from Yucatan. In The cenotes of Yucatan, A. S. Pearce, E. P. Creaser y F.G. Hall (eds.). Publication 457. Carnegie Institution of Washington, Washington, D.C. p. 153-155.

Erichson, W. F. 1837. Die Käfer der Mark Brandenburg vol. 1. F. H. Morin, Berlin. 740 p.

Fabricius, J. C. 1775. Systema Entomologiae. Libraria Korte, Flensburgi et Lipsiae. 832 p.

Fabricius, J. C. 1792. Entomologia Systematica, vol. 1, pars 1. C. G. Proft, Hafniae. 330 p.

Fall, H. C. 1901. List of the Coleoptera of Southern California, with notes on habits and distribution and descriptions on new species. Occasional Papers of the California Academy of Siences 8:1-282.

Fall, H. C. 1910. Micellaneous notes and descriptions of North American Coleoptera. Transactions of the American Entomological Society 36:89-197.

Fall, H. C. 1924. New species of North American Hydrobiini. Journal of the New York Entomological Society 32 85-89.

Fernández, L. A. y A. O. Bachmann. 1987. Revisión del género Helobata Bergroth (Coleoptera: Hydrophilidae). Revista de la Sociedad Entomológica Argentina 44:149-159.

Fikácek, M. y A. E .Z. Short. 2006. A revision of the Neotropical genus Motonerus Hansen (Coleoptera: Hydrophilidae: Sphaeridae). Zootaxa 1268:1-38.

Gentili, E. 1986a. I Laccobius americani-II. I1 genere Laccobius a sud del Canada (Coleoptera, Hydrophilidae). Annuario Osservatorio di Fisica Terrestre e Museo Antonio Stoppani del Seminario Arcivescoville di Milano 7(1984):31-40.

Gentili, E. 1986b. I Laccobius americani-III. I1 genere Laccobius a sud del Canada (Coleoptera, Hydrophilidae). Annuario Osservatorio di Fisica Terrestre e Museo Antonio Stoppani del Seminario Arcivescoville di Milano 8(1985):31-52.

Geoffroy, E. L. 1762. Histoire abrégée des insectes, vol. 1. Durand, Paris. 523 p.

Gundersen, R. W. 1977. New species and taxonomic changes in the genus Enochrus (Coleoptera: Hydrophilidae). The Coleopterists Bulletin 31:251-272.

Gundersen, R. W. 1978. Neartic Enochrus biology, key, descriptions and distribution (Coleoptera: Hydrophilidae). Departament of Biology Sciences, University of Minnesota, Minneapolis. 54 p. (publicación privada).

Hansen, M. 1989. New genera of Sphaeridiinae (Coleoptera: Hydrophilidae). Entomologica Scandinavica 20:251-262.

Hansen, M. 1991. The hydrophiloid beetles: phylogeny, classification and a revision of the genera (Coleoptera, Hydrophiloidea). Biologiske Skrifter, Det Kongelige Danske Videnskabernes Selskab 40:1-68.

Hansen, M. 1995. Evolution and classification of the Hydrophiloidea- a systematic review. In Biology, phylogeny, and classification of Coleoptera. Papers celebrating the $80^{\text {th }}$ birthday of Roy A. Crowson, vol. 1, J. Pakaluk y S. A.
Slipinski (eds.). Muzeum i Instytut Zoologii PAN, Varsovia. p. 321-353.

Hansen, M. 1999. Hydrophiloidea (s. str.) (Coleoptera). In World catalogue of insects, vol. 2, M. Hansen (ed.). Apollo, Copenhague. $416 \mathrm{p}$.

Hatch, M. H. 1965. The beetles of the Pacific Northwest, part IV: Macrodactyles, Palpicornes, and Heteromera. University of Washington Press, Seattle. 268 p.

Hendrich, J. S. 1975. A note on the occurrence of Sphaeridium scarabaeoides L. near Mexico City (Col. Hydropjilidsae). Coleopterists Bulletin 29:171.

Hinton, H. E.1933. Two coleopterous families new to Mexico. Pan-Pacific Entomologist 9:160-162.

Hinton, H. E. y H. L. Ancona. 1934. Fauna de coleópteros en nidos de hormigas (Atta), en México y Centro América. Anales del Instituto de Biología, Universidad Nacional Autónoma de México 5:243-248.

Horn, G. H. 1871. Descriptions of new Coleoptera of the United States, with notes on known species. Transactions of the American Entomological Society 3:325-344.

Horn, G. H. 1873. Revision of the genera and species of the tribe Hydrobiini. Proceedings of the American Philosophical Society 13:118-137.

Horn, G. H. 1890. Notes on some Hydrobiini of boreal America. Transactions of the American Entomological Society 17:237-278.

Horn, G. H. 1895. Coleoptera de Baja California (supplement I). Proceedings of the California Academy of Sciences 5:225-259.

Knisch, A. 1921. Die exotischen Hydrophiliden des Deutschen Entomologischen Museums (Col.). Archiv für Naturgeschichte 85 (1919), A:55-88.

Knisch, A. 1922. Hydrophiliden-studien. (Op. 10). Archiv für Naturgeschichte 88, A:87-126.

Knisch, A. 1924. Neue neotropische Palpicornier (Coleoptera: Hydrophilidae-Op.16). Wiener Entomologische Zeitung 41:114-140.

Kirsch, T. F. 1873. Beiträge zur Kenntniss der Peruanischen Käferfauna auf Dr. Abendroth's sammlungen basirt. Berliner Entomologische Zeitschrift 17:121-152.

Komarek, A. 2005. Taxonomic revision of Anacaena Thomson, 1859, II. Neotropical species (Coleoptera: Hydrophilidae). Koleopterologische Rundschau 75:253-301.

Lacordaire, T. 1854. Histoire naturelle des insectes. Genera des colóptères. 1. Librairie Encyclopédique de Roret, Paris. 486 p.

Lanzhu, J. y M. A. Jäch. 1998. Description of Eupotemus gen. nov. (Coleoptera: Epimetopidae) and world checklist of the species of Epimetopidae. Entomological Problems 29:95-97.

Latreille, P. A. 1802. Histoire naturelle, générale et particulière des crustacés et des insectes. Familles naturelles et generes, vol. 3. F. Dufart, Paris. 468 p.

Latreille, P. A. 1809. Genera crustaceorum et insectorum, 
secundum ordinem naturalem in familias disposita, iconibus exemplisque plurimis explicata, vol. 4. Amand Koenig, Paris. 399 p.

Lawrence, J. F. y A. F. Newton. 1995. Families and subfamilies of Coleoptera (with selected genera, notes, references and data on family-group names). In Biology, phylogeny, and classification of Coleoptera. Papers celebrating the $80^{\text {th }}$ birthday of Roy A. Crowson, vol I., P. Pakaluk y S. A. Slipinski (eds.). Muzeum i Instytut Zoologii PAN, Varsovia. p. 779-1006.

Leach, W. E. 1815. Entomology. In Edinburgh encyclopaedia, vol. 9, D. Brewster (ed.). Balfour, Edinburgh. p. 57-172.

Leach, W. E. 1817. The zoological miscellany, vol. 3. R. P. Nodder, London. 151 p.

LeConte, J. L. 1852. Descriptions of new species of Coleoptera, from California. Annals of the Lyceum of Natural History of New York 5:185-216.

LeConte|, J. L. 1855. Synopsis of the Hydrophilidae of the United States. Proceedings of the Academy of Natural Sciences of Philadelphia 7:356- 375.

Leconte, J. L. 1861. New species of Coleoptera inhabiting the Pacific district of the United States. Proceedings of the Academy of Natural Sciences of Philadelphia 13:338- 359.

Leconte, J. L. 1866. Additions to the coleopterous fauna of the United States. No. 1. Proceedings of the Academy of Natural Sciences of Philadelphia (1866):361-394.

LeConte|, J. L. 1874. Descriptions of new Coleoptera chiefly from the Pacific slope of North America. Transactions of the American Entomological Society 5:43-72.

Leech, H. B. 1945. A few additions and corrections to R.E. Blackwelder's "Checklist of the coleopterous insects of Mexico, Central America, the West Indies, and South America. Part. I". Entomological News 55 10:266-268.

Leech, H. B. 1948a. Some Nearctic species of palpicorn water beetles, new and old (Coleoptera: Hydrophilidae). Wasmann Collector 7:33-46.

Leech, H. B. 1948b. Contributions toward a knowledge of the insect fauna of Lower California 11. Coleoptera: Haliplidae, Dytiscidae, Gyrinidae, Hydrophilidae, Limnebiidae. Proceedings of the California Academy of Science 24:375-483.

Leech, H. B. y H. P. Chandley. 1956. Aquatic Coleoptera. In Aquatic insects of California, R. L. Usinger, (ed.). University of California Press, Berkeley. p. 293-371.

Linnaeus, C. 1758. Systema Naturae...(ed. 10), vol. 1. Laur. Salvii, Holmiae. 284 p.

Makhan, D. 1994. Thirty-five new Hydrochus species from the Old and New World (Coleoptera: Hydrophilidae). Annales Historico-Naturales Musei Nationalis Hungarici 86:29-42.

Mannerheim, C. G. 1852. Zweiter Nachtrag zur Kaefer-Fauna der Nord-Amerikanaischen Laender des Russischen Reiches. Bulletin de la Société Impériale des Naturalistes de Moscou
25:283-387.

Mannerheim, C. G. 1853. Dritter Nachtrag zur Kaefer-Fauna der Nord-Amerikanischen Laender des Russischen Reiches. Bulletin de la Société Impériale des Naturalistes de Moscou 26:95-273.

Márquez-Luna, J. y J. L. Navarrete-Heredia. 1995. Biological notes on Oosternum attacomis (Coleoptera: Hydrophilidae), from Morelos State, Mexico. Entomological News 106:203-208.

Melsheimer, F. E. 1844. Descriptions of new species of Coleoptera of the United States. Proceedings of the Academy of Natural Sciences of Philadelphia 2:98-118.

Miller, C. D. 1974. Revision of the new world Chaetarthria (Coleoptera: Hydrophilidae). Entomological Americana 49:1-120.

Motschulsky, V. 1859. Coléoptères nouveaux de la Californie. Bulletin de la Société Impériale des Naturalistes de Moscou 32:122-185, 357-410.

Mulsant, E. 1844a. Histoire naturelle des coléoptères de France. Palpicornies. L. Maison, Paris; Ch. Savy Jeune, Lyon. 196 p.

Mulsant, E. 1844b. Description de quelques palpicornes inédits. Annales de la Société d'Agriculture de Lyon 7:372-382.

Navarrete-Heredia, J. L. 1992. Primer registro de Cymbiodyta brevipalpis pygmea (Coleoptera: Hydrophilidae) para Morelos. Anales del Instituto de Biología, Universidad Nacional Autónoma de México Serie Zoología 63:279-280.

Nelson, F. R. S. 1977. Predation on mosquito larvae by beetles larvae, Hydrophilus triangularis and Dytiscus marginalis. Mosquito News 37:628-630.

Newton, A. F. y M. K. Thayer. 1992. Current classification and family-group names in Staphyliniformia (Coleoptera). Fieldiana: Zoology, N. S. 67:1-92.

Oliva, A., L. A. Fernández y A. O. Bachmann. 2002. Sinopsis de los Hydrophiloidea acuáticos de la Argentina (Insecta: Coleoptera). Monografías de Museo Argentino de Ciencias Naturales 2:1-67.

Orchymont, A. d'. 1921. Le genere Tropisternus. I. (Coleoptera: Hydrophilidae). Annales de la Société Entomologique de Belgique 61:349-374.

Orchymont, A. d'. 1938. Contribution à l'étude des Palpicornia XII. Bulletin et Annales de la Société Entomologique de Belgique 78:426-438.

Orchymont, A. d'. 1942. Revision des Laccobius américains (Coleoptera: Hydrophilinae, Hydrobiini). Bulletin du Musée Royal d'Histoire Naturelle de Belgique 18:1-18.

Orchymont, A. d'. 1946. Notes on some American Berosus (s.str.) (Coleoptera- Palpicornia-Hydrophilidae). Bulletin du Musée Royal d'Histoire Naturelle de Belgique 22:1-20.

Pennak, R. W. 1978. Fresh-water invertebrates of the United States. Wiley, New York. 803 p.

Perkins, P. D. 1979. Six new neotropical species of aquatic beetles in the Epimetopus costatus complex (Coleoptera: 
Hydrophilidae). The Coleopterists Bulletin 33:319-325.

Perkins, P. D. 1980. Three new Middle America species of aquatic beetles in the genus Notionotus Spangler (Hydrophilidae: Hydrobiinae). Journal of the New York Entomological Society 87:304-311.

Régimbart, M. 1901. Revision des grands Hydrophiles. Annales de la Société entomologique de France 70:188-232; supplement:665-666.

Santiago-Fragoso, S y L. Vázquez-Navarrete, 1989. Coleópteros acuáticos y semiacuáticos del río Amacuzac (Huajintlan y El Estudiante) Morelos, México. Anales del Instituto de Biología, Universidad Nacional Autónoma de México, Serie Zoología. 60:405-426.

Santiago-Fragoso, S. y E. Mejorada-Gómez. 1995. Anew scaraveger beetle, Enochrus spangleri (Coleoptera: Hydrophilidae), from Mexico. Entomological News 106:36-38.

Santiago-Fragoso, S. y E. Mejorada-Gómez 1999. Aquatic Coleoptera from Lake Xochimilco, México. Entomological News 110:302-310.

Santiago-Fragoso, S. y J. C. Sandoval-Manrique. 2001. Coleópteros acuáticos y su relación con la dinámica fisicoquímica del río Cuautla (tramo TetelcingoAnenecuilco), Morelos, México. Hidrobiologia 11:19-30.

Say, T. 1823. Descriptions of coleopterous insects collected in the late Expedition to the Rocky Mountains, performed by order of Mr. Calhoun, Secretary of War, under the command of Major Long. Journal of the Academy of Natural Sciences of Philadelphia 3:139-216.

Say, T. 1825. Descriptions of new species of Coleopterous Insects inhabiting the United States. Journal of the Academy of Natural Sciences of Philadelphia 5:160-204.

Say, T. 1835. Descriptions of new North American Coleopterous Insects, and observations on some already described. Boston Journal of Natural History 1:151-203.

Schwarz, E. A. y H. S. Barber. 1918. Two new hydrophilid beetles. Proceeding of the Entomological Society of Washington 19:129-135.

Sharp, D. 1879. On some Coleoptera from the Hawaiian Islands. Transactions of the Entomological Society of London (1879):77-105.

Sharp, D. 1882. Insecta Coleoptera, vol. 1, part 2. In Biologia Centrali-Americana (16) xv + 824 p., F. D. Godman y O. Salvin (eds.). London. p.144

Sharp, D. 1887. Insecta Coleoptera, vol. 1, part 2. In Biologia Centrali-Americana (16) xv + 824 p., F. D. Godman y O. Salvin (eds.). London. p. i-xi, 673-824.

Short, A. E. Z. 2005a. Two new species of Enochrus Thomson, subgenus Hugoscottia Knisch, from Costa Rica y Mexico (Coleoptera Hydrophylidae). Zootaxa 865:1-7.

Short, A. E. Z. 2005b. A review of the subtribe Acidocerina of Central America with special reference to Costa Rica. Koleopterologische Rundschau 75:191-226.
Short, A. E. Z. y P. D. Perkins. 2004. A revision of the Oocyclus Sharp of México y Centroamérica (Coleoptera: Hydrophilidae). Zootaxa 783:45.

Short, A. E. Z. y P. J. Torres. 2006. Review of the Hemiosus Sharp and Derallus Sharp in North America, Central America and the Caribbean (Coleoptera: Hydrophilidae: Berosini). Zootaxa 1369:1-17.

Short, A. E. Z. y F. Hebauer. 2006. World catalogue of Hydrophiloidea additions and corrections, 1 (1999-2005) (Coleoptera), Koleopterologische Rundschau 76:315-359.

Smetana, A. 1974. Revision of the genus Cymbiodyta Bedel (Coleoptrera: Hydrophilidae). Memoirs of the Entomological Society of Canada 93:1-113.

Smetana, A. 1975. Revision of the New World genera of the tribe Omicrini trib. nov. of hydrophilid subfamily Sphaeridiinae (Coleoptera). Studies on Neotropical Fauna 10:153-182.

Smetana, A. 1978. Revision of the subfamily Sphaeridiinae of America north of Mexico (Coleoptera: Hydrophilidae) Memoirs of the Entomological Society of Canada 105:1-290.

Smetana, A. 1980. Revision of the genus Hydrochara Berthold (Coleoptera: Hydrophilidae). Memoirs of the Entomological Society of Canada 111:1-100.

Smetana, A. 1984. Revision of the subfamily Sphaeridiinae of America north of Mexico (Coleoptera: Hydrophilidae). Supplementum 2. Canadian Entomologist 116:555-566.

Smetana, A. 1985a. Revision of the subfamily Helophorinae of the Nearctic region (Coleoptera: Hydrophilidae). Memoirs of the Entomological Society of Canada 131:1-154.

Smetana, A. 1985b. Synonymical notes on Hydrophilidae (Coleoptera). Coleopterists Bulletin 39:328.

Smetana, A. 1988. Review of the family Hydrophilidae of Canada and Alaska (Coleoptera). Memoirs of the Entomological Society of Canada 142:1-316.

Smetana, A. 1990. Type species designation for Aculomicrus Smetana (Coleoptera: Hydrophilidae). Coleopterists Bulletin 44:64.

Solier, A. J. J. 1834. Observations sur la tribu des Hydrophiliens, et principalment sur le genre Hydrophilus de Fabricius. Annales de la Société Entomologique de France 3:299-318.

Spangler, P. J. 1960. A revision of the genus Tropisternus (Coleoptera: Hydrophilidae). Ph. D. Thesis, Faculty of the Graduate School, University of Missouri, Columbia. 365 p.

Spangler, P. J. 1962. A new species of the genus Oosternum and a key to the U.S. (Coleoptera: Hydrophilidae). Proceedings of the Biological Society of Washington 75:97-100.

Spangler, P. J. 1972. A new genus and two new species of madicolous beetles from Venezuela (Coleoptera: Hydrophilidae). Proceedings of the Biological Society of Washington 85:139-146.

Spangler, P. J 1982. Coleoptera. In Aquatic biota of Mexico, Central America and West Indies, S. H. Hurlbert y A. 
Villalobos-Figueroa (eds.).University of San Diego, California. p. 323-395.

Spangler, P. J. 1991. Hydrophilidae (Hydrophiloidea) (Including Helophoridae, Hydrochidae, Sphaeridiidae, Spercheidae) In: Immature insects, vol. 2, F. W. Stehr (ed.) Kendall-Hunt, Dubuque, Iowa. p. 355-359.

Spangler P. J. y S. Santiago-Fragoso. 1979. A new water scavenger beetle from Mexico (Coleoptera: Hydrophilidae). Proceedings of the Biological Society of Washington 92:333-338.

Spangler, P. J. y H. A. Huacuja. 1982. Deltostethus scitulus, a new hydrophilid beetle from Mexico (Coleoptera: Hydrophilidae). Entomological News 93:1-7.

Stephens, J. F. 1835 (1832-1835). Illustrations of British entomology. Mandibulata, vol. 5. Baldwin and Cradock, London. 488 p.

Thomson, C. G. 1859. Skandinaviens Coleoptera, vol. 1. Berlingska Boktryckeriet, Lund. 290 p.

Thomson, C. G. 1867. Skandinaviens Coleoptera, vol. 9. Lundbergska Boktryckeriet, Lund. 407 p.

Van Tassell, E. 1963. A new Berosus from Arizona, with a key to the Arizona species (Coleoptera, Hydrophilidae). Coleopterists Bulletin 17:1-5.

Van Tassell E. 1966. Taxonomy and biology of the subfamily Berosinae of North and Central America and the West Indies (Coleoptera: Hydrophilidae). Ph. D. thesis, The Catholic University of America, Washington, D. C. 329 p.

Van Tassell, R. E. 2000. Family Hydrophilidae. In American beetles, vol. 1 (Archostemata, Myxophaga, Adephaga, Polyphaga: Staphyliniformia), R. A. Arnett y M. C. Thomas (eds.). CRC, Boca Raton, Florida. p. 187-208.

White, D. S. y W. V. Brigham. 1996. Aquatic Coleoptera. In An introduction to the aquatic insects of North America, R. W. Merritt y K.W. Cummins (eds.). Kendall-Hunt, Iowa. p. 399-473.

Wollaston, T. V. 1854. Insecta Maderensia; being an account of the insects of the islands of the Maderian group. J. v. Voorst, London. $43+634$ p., 13 pl.

Wooldridge D. P. 1966. Notes on Nearctic Paracymus with descriptions of new species (Coleoptera: Hydrophilidae). Journal of Kansas Entomological Society 39:712-725.

Wooldridge D. P. 1969. New species of Paracymus from Mexico and Central America. Journal of Kansas Entomological Society 42:413-421.

Wooldridge D. P. 1973. Two new Mexican Paracymus (Coleoptera: Hydrophilidae). Journal of Kansas Entomological Society 46:434-436.

Wooldridge D. P. 1975. Paracymus securus, a new species from Baja California (Coleoptera: Hydrophilidae). Journal of Kansas Entomological Society 48:1-83.

Young, F. N. 1954. The water beetles of Florida. University of Florida. Studies. Biological Science Series 5:1-238.

Zaitzev, F. A.1908. Catalogue des coléoptères aquatiques des familles Dryopidae, Georyssidae, Cyathoceridae, Heteroceridae et Hydrophilidae. Horae Societatis Entomologicae Rossicae 38:283-420. 


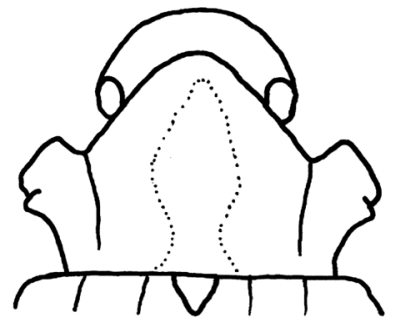

1
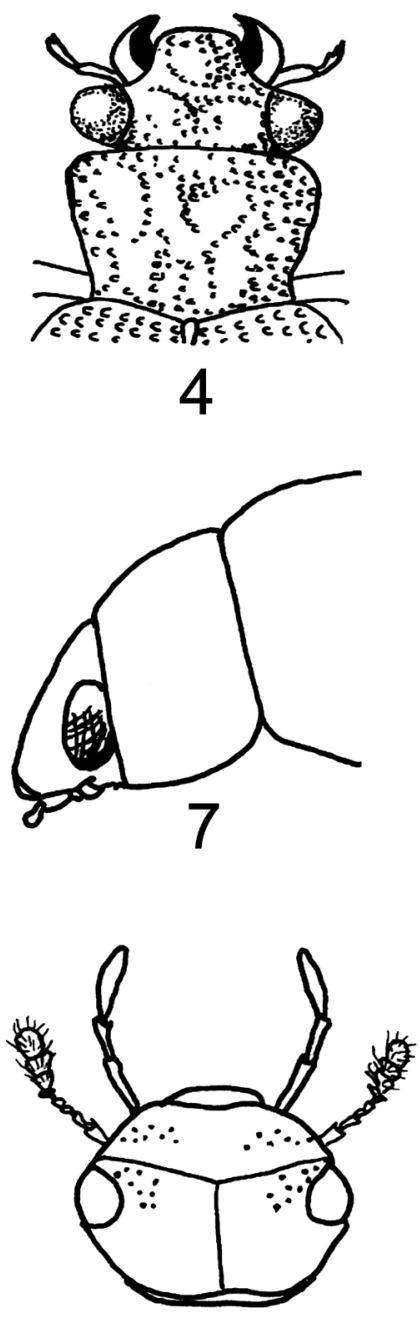

10
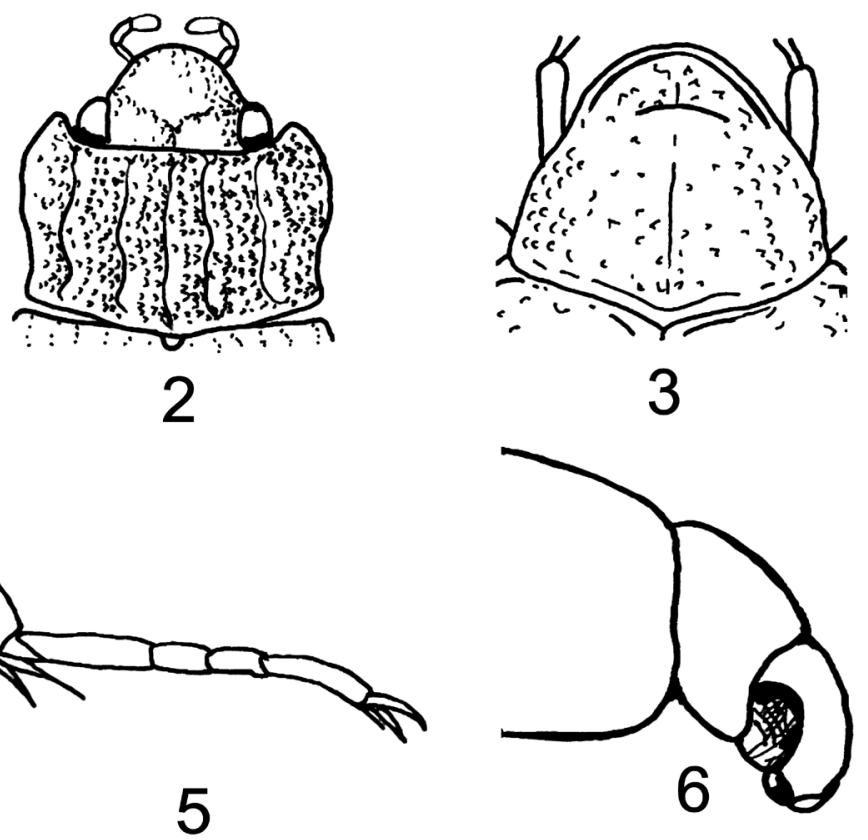

5

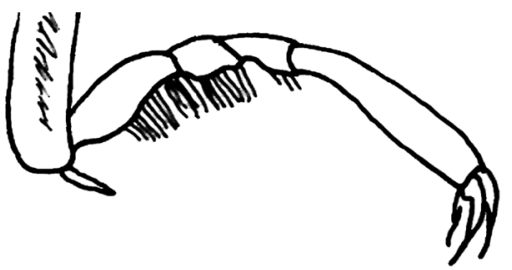

8

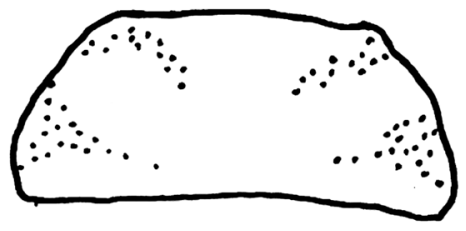

11

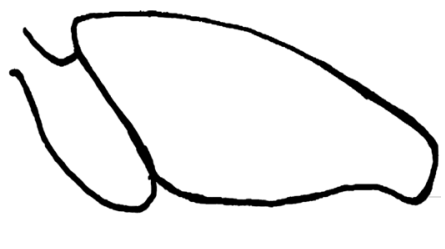

9

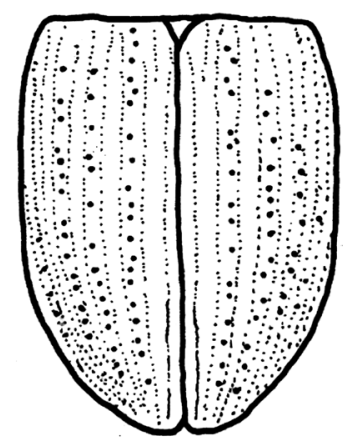

12

Figuras 1-12. Detalles de la morfología de Hydrophiloidae. 1, vista dorsal del pronoto Epimetopus sp.; 2, vista dorsal del pronoto de Helophorus sp.; 3, vista dorsal del pronoto Georissus sp. 4; vista dorsal del pronoto de Hydrochus sp.; 5, tarsómero posterior de Berosus sp.; 6, vista lateral de la cabeza de Berosus sp.; 7, vista lateral de la cabeza de Laccobius sp.; 8, protarso del macho de Berosus sp.; 9, trocanter posterior de Laccobius sp.; 10, series de puntos sobre la cabeza de Hydrophilini; 11, series de puntos sobre el pronoto de Hydrophilini; 12, series de puntos sobre los élitros de Hydrophilini. (Las figuras que se indican fueron tomadas y modificadas de Hansen, 1999 (9); Van Tassell, 2000 (5-8, 9-12) y White y Brigham, 1996 (1-4). 


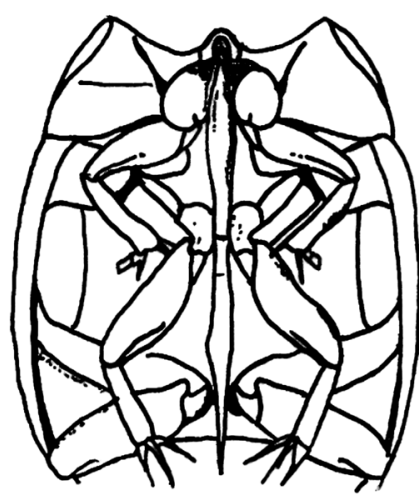

13

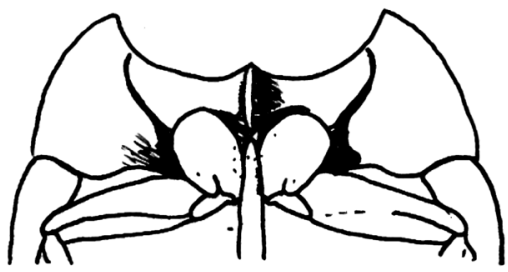

14

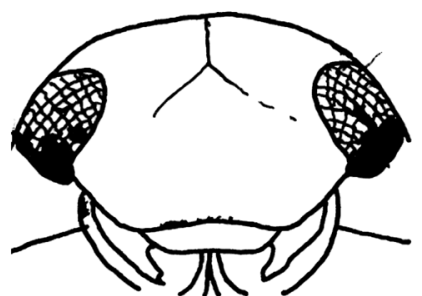

15

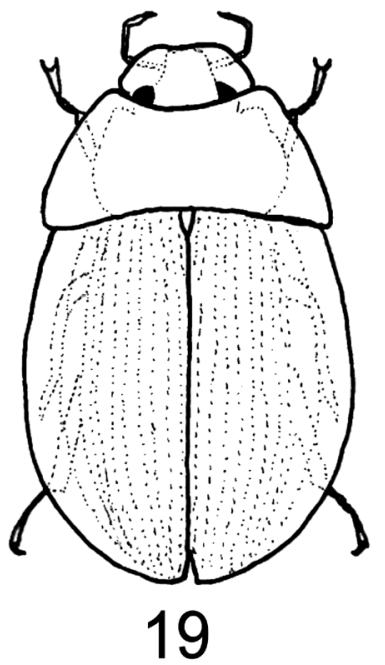

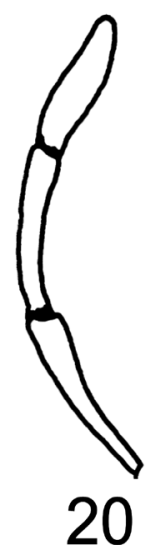

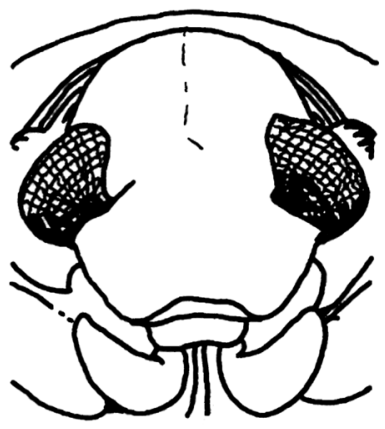

17

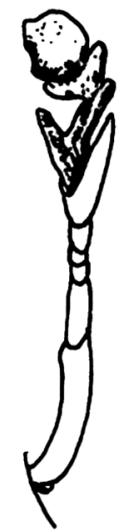

18

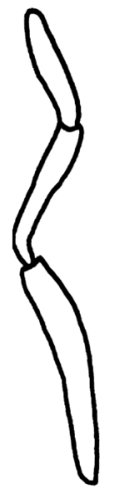

21

22

Figuras 13-22. Detalles de la morfología de Hydrophiloidae. 13, prosternón y quilla media en Hydrophilus triangularis; 14, prosternón carinado en Hydrocara sp.; 15, margen frontal del clípeo en Hydrocara sp.; 16, maza antenal de Hydrocara sp.; 17, margen frontal del clípeo en Hydrobiomorpha sp.; 18, maza antenal de Hydrobiomorpha sp.; 19, vista dorsal de Helobata sp.; 20, palpos maxilares de Cymbiodyta sp.; 21, mesoesternón con una cresta transversa en Cymbiodyta sp.; 22, palpos maxilares de Enochrus sp. (Las figuras que se indican fueron tomadas y modificadas de Van Tassell, 2000 (13-18, 20-22) y White y Brigham, 1996 (19). 


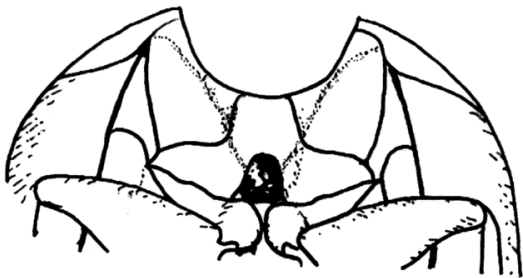

23
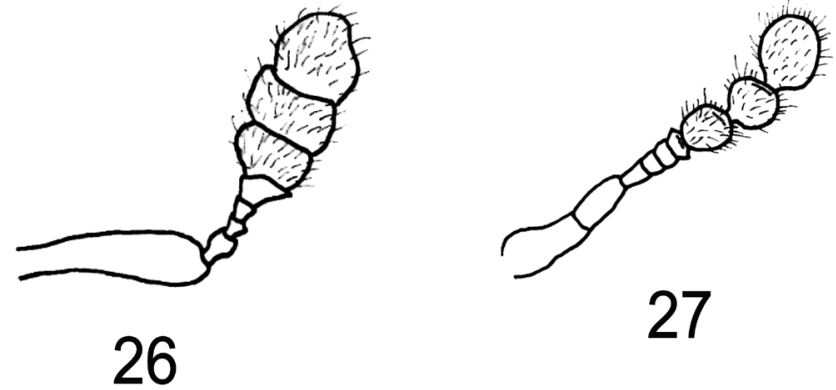

27

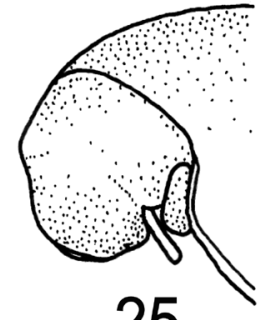

25

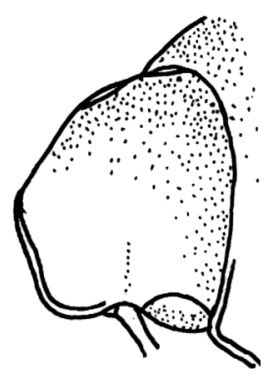

28

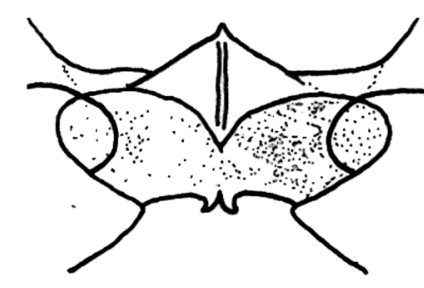

29

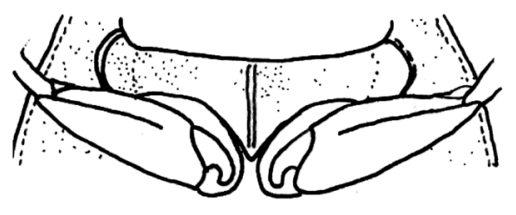

32

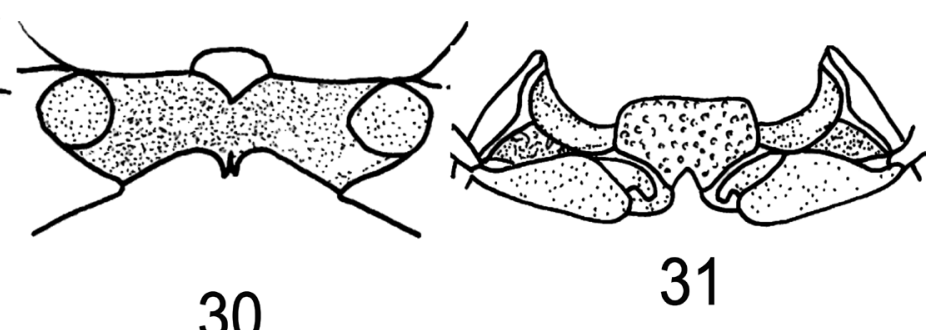

30

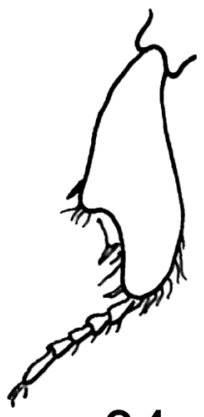

33

34

Figuras 23-34. Detalles de la morfología de Hydrophiloidae. 23, mesosternón de Anacaena sp.; 24, vista dorsolateral de la cabeza de Sphaeridium scarabaeoides; 25, vista dorsolateral de la cabeza de Psalitrus sp.; 26 maza antenal compacta en Sphaeridium sp.; 27, maza antenal no compacta en Phaenonotum sp.; 28, vista dorsolateral de la cabeza de Megasternini; 29, prosternón visible enfrente de las procoxas en Heteryon sp.; 30, prosternón poco visible enfrente de las procoxas en Aculomicrus sp.; 31, prosternón con largos y profundos surcos antenales que rebasan el margen lateral del protórax en Cryptopleurum sp.; 32, prosternón con surcos antenales que no rebasan el margen lateral del protórax en Cercyon sp.; 33, líneas femorales del metasternón completas en Cryptopleurum sp.; 34, margen externo de las tibias anteriores fuertemente escotado en Megasternum sp. (Las figuras que se indican fueron tomadas y modificadas de Hansen, 1999 (24, 25, 28-33) y Van Tassell, 2000 (23, 26, 27 y 34). 

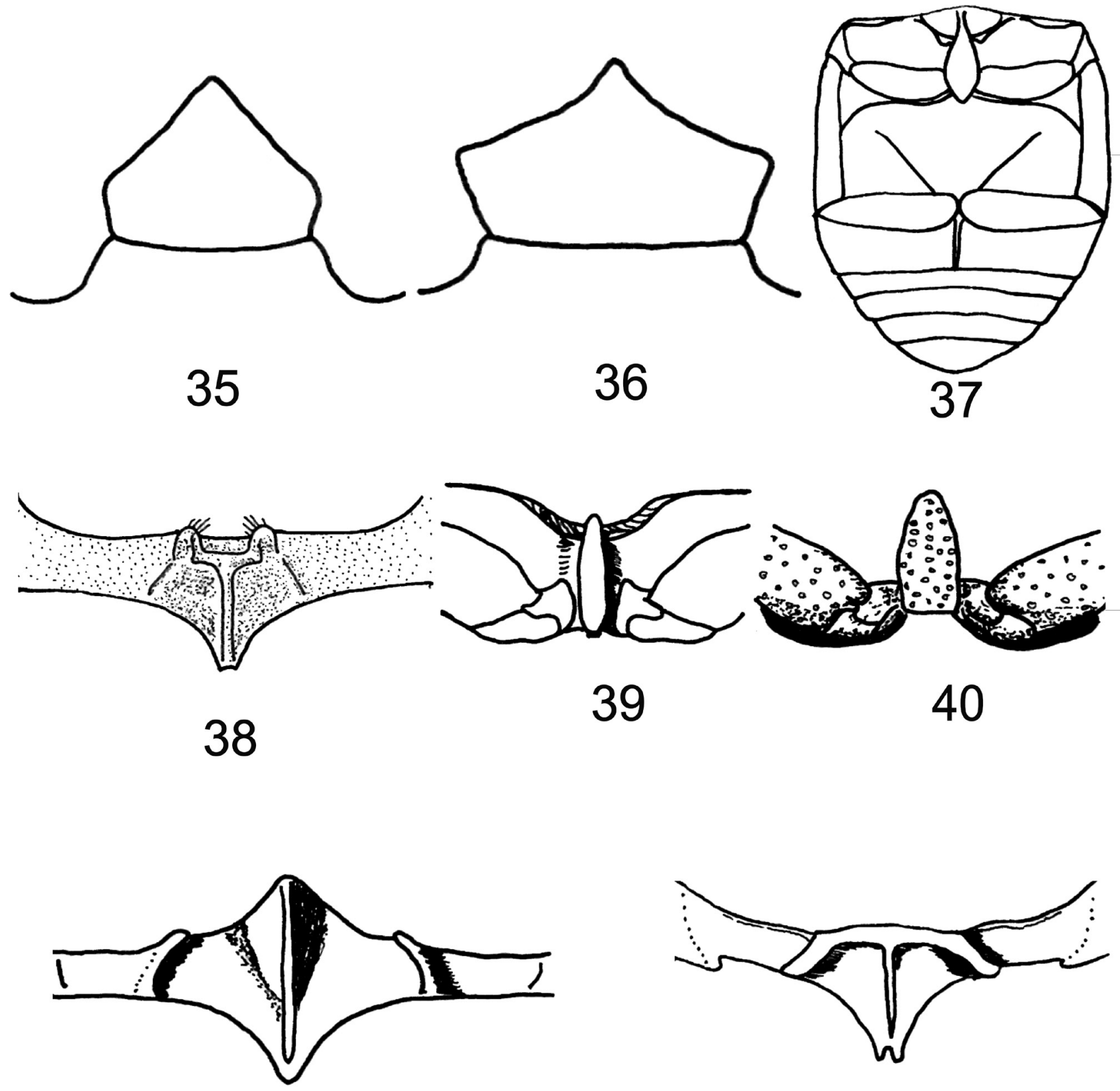

41

42

Figuras 35-42. Detalles de la morfología de Hydrophiloidae. 35, placa mesoesternal ancha como larga en Pachysternum sp.; 36, placa mesoesternal más ancha que larga en Cryptopleurum sp.; 37, esquinas anterolaterales del metaesterno claramente demarcadas del resto del metaesternón por un reborde arqueado en Oosternum sp.; 38, margen anterior del prosternón con una incisión subrectangular media, cuyos márgenes laterales son rebordes proyectados en Motonerus sp.; 39, placa mesoesternal de alargada a ovalada o casi lineal en Cercyon sp.; 40, placa mesoesternal 2 o 3 veces más larga que ancha en Pelosoma sp.; 41, el centro del prosternón tectiforme y fuertemente carinado a la mitad en Agna sp.; 42, el centro del prosternón plano o finamente carinado en Deltostetus sp. (Las figuras que se indican fueron tomadas y modificadas de Hansen, 1999 (35-38) y Van Tassell, 2000 (39-42). 


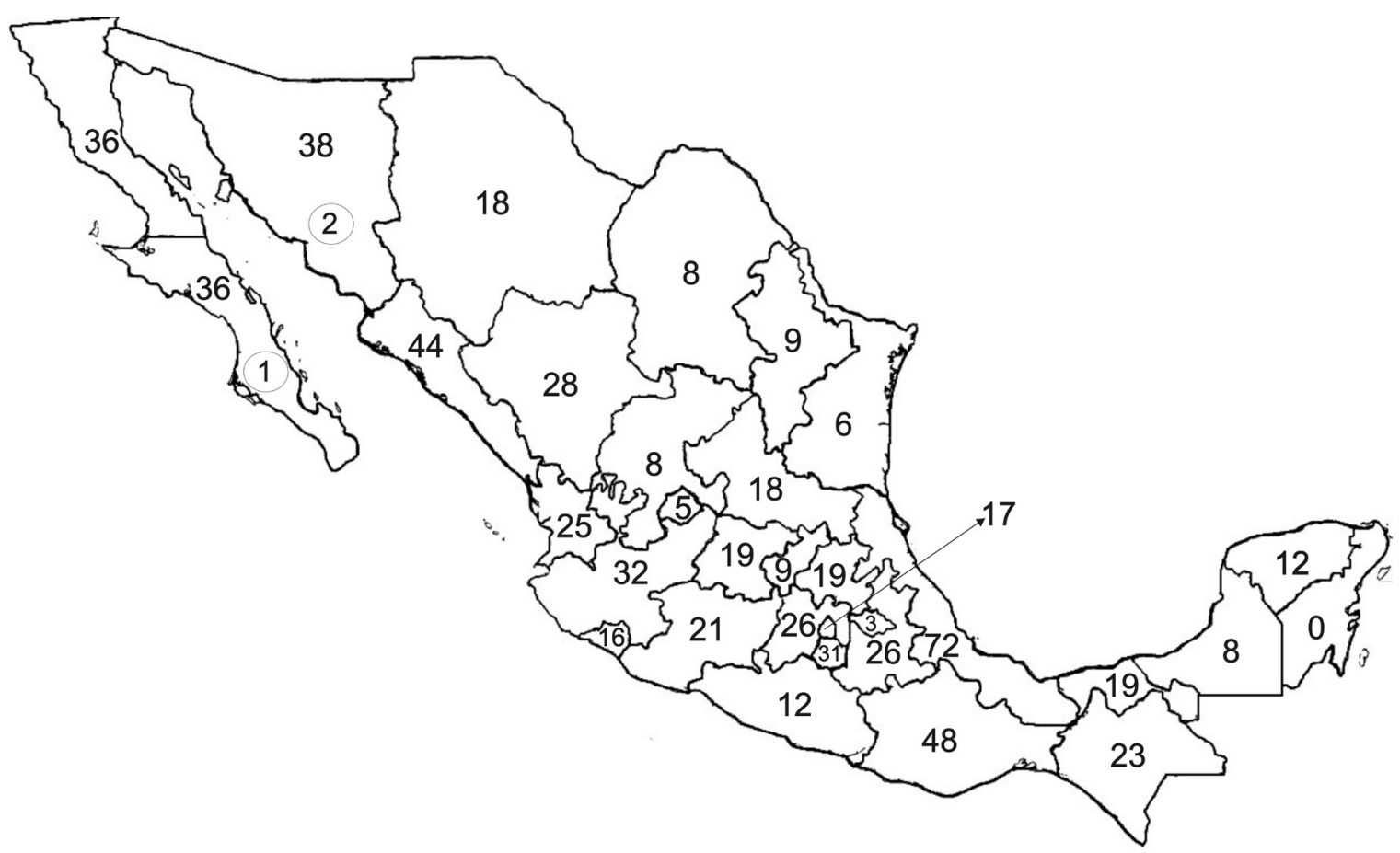

Figura 43. Número de especies por estado de las familias Hydrophilidae (sólo cifras) y Epimetopidae (dentro de un círculo) en México.

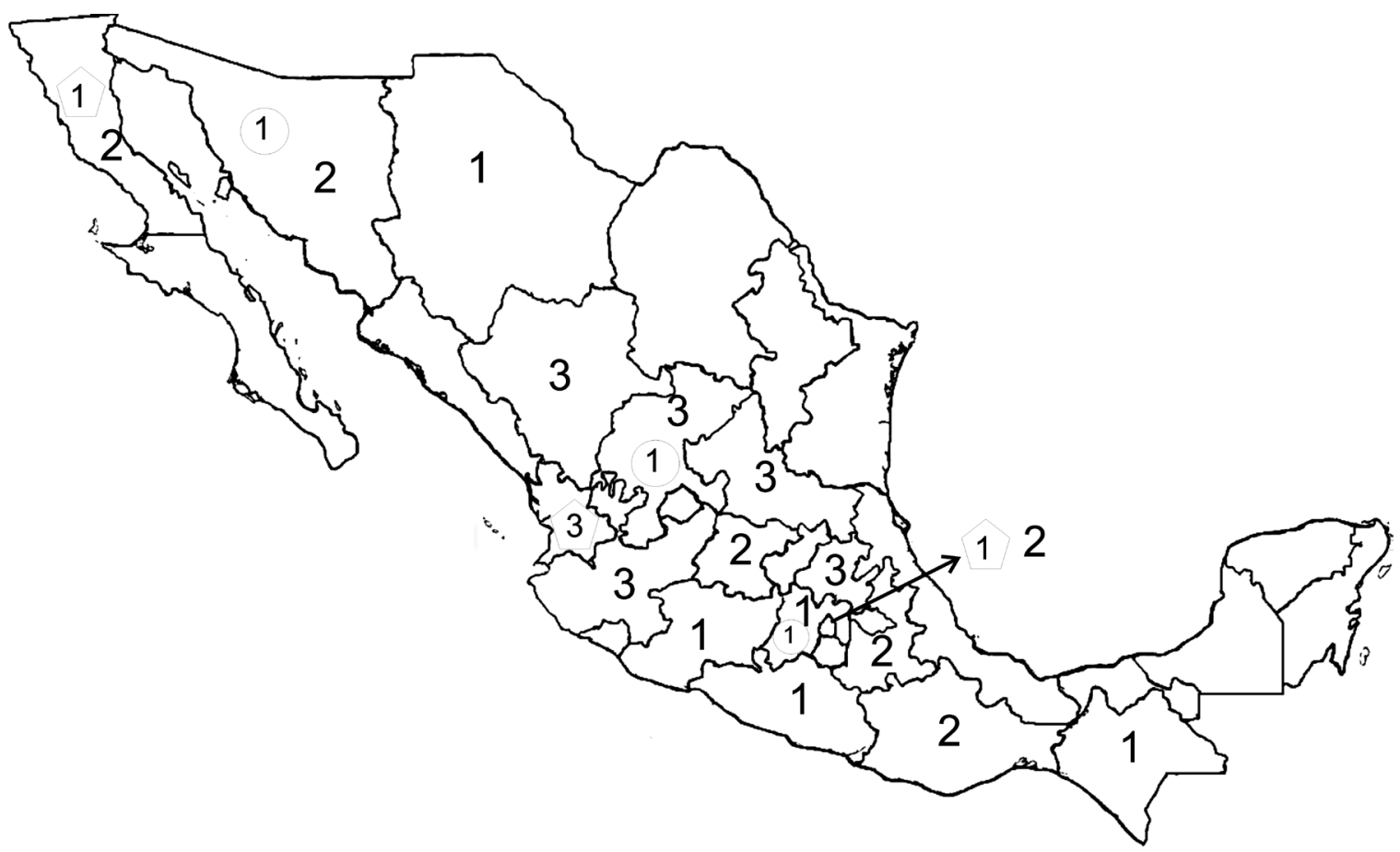

Figura 44. Número de especies por estado de las familias Helophoridae (sólo cifras), Georissidae (dentro de un círculo) e Hydrochidae (dentro de un pentágono) en México. 